\title{
Quantitative Regularity for $p$-Minimizing Maps Through a Reifenberg Theorem
}

\author{
Mattia Vedovato ${ }^{1}$ \\ Received: 7 October 2019 / Accepted: 10 December 2020 / Published online: 12 January 2021 \\ (C) The Author(s) 2021
}

\begin{abstract}
In this article we extend to arbitrary $p$-energy minimizing maps between Riemannian manifolds a regularity result which is known to hold in the case $p=2$. We first show that the set of singular points of such a map can be quantitatively stratified: we classify singular points based on the number of almost-symmetries of the map around them, as done in Cheeger and Naber (Commun Pure Appl Math 66(6): 965-990, 2013). Then, adapting the work of Naber and Valtorta (Ann Math (2) 185(1): 131-227, 2017), we apply a Reifenberg-type Theorem to each quantitative stratum; through this, we achieve an upper bound on the Minkowski content of the singular set, and we prove it is $k$-rectifiable for a $k$ which only depends on $p$ and the dimension of the domain.
\end{abstract}

Keywords Harmonic maps · Quantitative stratifications · Volume estimates ·

Regularity

Mathematics Subject Classification 35B65 $\cdot 53 \mathrm{C} 43 \cdot 35 \mathrm{~J} 92$

\section{Introduction}

The aim of this article is to study the singular set $\mathcal{S}(u)$ of maps that take values in a smooth Riemannian manifold and minimize the $p$-energy functional:

$$
\mathcal{E}_{p}(u) \doteq \int|\nabla u|^{p}
$$

for $p \in(1, \infty)$; we assume that $u: B \subset \mathcal{M} \rightarrow \mathcal{N}$, where $B$ is a geodesic ball in the smooth Riemannian manifold $\mathcal{M}$, and $\mathcal{N}$ is a smooth closed Riemannian manifold.

Mattia Vedovato

mattia.vedovato@unitn.it

1 Università degli Studi di Trento, Trento, Italy 
Here by singular set we mean the set of points where the map is not continuous. The information we obtain is twofold:

1. First we obtain a Minkowski-type estimate on the singular set: if $u: B \subset \mathcal{M} \rightarrow \mathcal{N}$, and the total $p$-energy of $u$ is bounded by $\Lambda$, then

$$
\operatorname{Vol}\left(\mathcal{B}_{r}(\mathcal{S}(u)) \cap B^{\prime}\right) \leq C(\mathcal{M}, \mathcal{N}, \Lambda, p) r^{\lfloor p\rfloor+1}
$$

where $B^{\prime} \subset \subset B$ is a smaller geodesic ball and $\mathcal{B}_{r}(\mathcal{S}(u))$ is the $r$-tubular neighborhood of $\mathcal{S}(u)$. In particular, the Minkowski dimension of $\mathcal{S}(u)$ is at most $\operatorname{dim} \mathcal{M}-\lfloor p\rfloor-1$, and the upper Minkowski content (in $B^{\prime}$ ) is bounded by a universal constant $C(\mathcal{M}, \mathcal{N}, \Lambda, p)$.

2. Secondly, a rectifiability result is proved: we show that $\mathcal{S}(u)$ is $(\operatorname{dim} \mathcal{M}-\lfloor p\rfloor-1)$ rectifiable.

In what follows, we set $m \doteq \operatorname{dim} \mathcal{M}$. Our results complete the following scheme:

- $p=2$ : in 1982, Schoen and Uhlenbeck proved through a dimension reduction argument that any 2-energy minimizing map is $\mathcal{C}^{0, \alpha}$ outside of a set of Hausdorff dimension at most $m-3$ (see [19]). Furthermore, by standard elliptic regularity, the regularity outside the singular set can be improved to $\mathcal{C}^{\infty}$.

- $p \in(1, \infty)$ : in 1987, Schoen and Uhlenbeck's result was extended to $p$-harmonic maps by Hardt and Lin [6]. The best regularity one can achieve outside the singular set is $\mathcal{C}^{1, \alpha}$; so any $p$-energy minimizing map is $\mathcal{C}^{1, \alpha}$ outside of a set of Hausdorff dimension at most $m-\lfloor p\rfloor-1$. Notice that the case $m \leq p$ was already completely solved here: in this case, there are no singular points, and the map is everywhere $\mathcal{C}^{1, \alpha}$. The only case worth studying is $m>p$.

- $p=2$ : in 2013, Cheeger and Naber [2] proved that the singular set of a 2minimizing map with energy bounded by $\Lambda$ satisfies the following estimate:

$$
\operatorname{Vol}\left(\mathcal{B}_{r}(\mathcal{S}(u)) \cap B^{\prime}\right) \leq C(\mathcal{M}, \mathcal{N}, \Lambda, \varepsilon) r^{3-\varepsilon}
$$

for any $\varepsilon>0$; it is assumed that the dimension of the domain is at least 3 . This implies that the Minkowski dimension of $\mathcal{S}(u)$ is at most $m-3$, but gives no bound on the Minkowski content. Here a notion of "quantitative stratification" of the singular set was introduced, and the result was obtained through a relatively simple covering of each singular stratum (and by making explicit the link between singular set and stratification).

- $p \in(1, \infty)$ : in 2014, Naber, Valtorta and Veronelli [15] extended the estimate (1.2) to $p$-minimizing maps: they showed that in this case

$$
\operatorname{Vol}\left(\mathcal{B}_{r}(\mathcal{S}(u)) \cap B^{\prime}\right) \leq C(\mathcal{M}, \mathcal{N}, \Lambda, p, \varepsilon) r^{\lfloor p\rfloor+1-\varepsilon}
$$

for any $\varepsilon>0$. It is assumed that the dimension of the domain is greater than $p$ : we have already noticed, however, that this is the only interesting case.

- $p=2$ : in 2017, Naber and Valtorta [13] improved the estimate (1.2) for 2minimizing maps, removing the dependence on the parameter $\varepsilon$ : assuming that 
$m>2$, then the singular set of a 2-minimizing map with energy bounded by $\Lambda$ satisfies

$$
\operatorname{Vol}\left(\mathcal{B}_{r}(\mathcal{S}(u)) \cap B^{\prime}\right) \leq C(\mathcal{M}, \mathcal{N}, \Lambda) r^{3}
$$

and thus the upper Minkowski content of $\mathcal{S}(u)$ is bounded by a constant $C$. Moreover, in the same article they showed that $\mathcal{S}(u)$ is actually $(m-3)$-rectifiable. The main idea to prove both the Minkowski estimate and rectifiability was to replace the simple covering argument of [2] with a more refined one, which makes use of a suitable version of Reifenberg Theorem.

In this article, we adopt the same technique used in [13]: after giving an appropriate definition of singular stratification (adapted to the case of $p$-minimizing maps), we exploit the very same version of Reifenberg Theorem developed in [13, Theorems 3.3 and 3.4] to build a controlled covering of each stratum. Notice that analogous results (still exploiting this technique) are available for approximate harmonic maps [14] and for $Q$-valued energy minimizers [7].

\subsection{Definitions and Notation}

From now on, $\mathcal{N}$ will always be an $n$-dimensional compact, smooth Riemannian manifold without boundary, while $\mathcal{M}$ is a $m$-dimensional smooth Riemannian manifold. For convenience, we can assume $\mathcal{N}$ is isometrically embedded in a Euclidean space $\mathbb{R}^{N}$ - thanks to the well known Nash embedding Theorem (see [12]). For any $p \in(1, \infty)$, one can then define the Sobolev space $W^{1, p}(\mathcal{M}, \mathcal{N})$ as the space of maps $u \in W^{1, p}\left(\mathcal{M}, \mathbb{R}^{N}\right)$ such that $u(x) \in \mathcal{N}$ for almost every $x \in \mathcal{M}$.

Remark (Relation between $p$ and $m$ ) If $p>m$, by the Sobolev embedding theorem one has that $W^{1, p}(\mathcal{M}, \mathcal{N}) \hookrightarrow \mathcal{C}^{0}(\mathcal{M}, \mathcal{N})$. Consequently, this case is not particularly interesting for our purposes, since the singular set is empty. More generally, in the case $\lfloor p\rfloor \geq m$ (thus also when $p$ is an integer and $p=m$ ), it is a known fact that $p$-minimizers have no singular points (see for example [15, Theorem 2.19]). Thus, from now on, we will implicitly assume $p<m$.

The aim of this paper is to study the singular set of minimizers of the $p$-energy. We now give the main definitions involved.

Definition 1.1 ( $p$-energy) If $u \in W^{1, p}(\mathcal{M}, \mathcal{N})$, with $(\mathcal{M}, g)$ Riemannian manifold of dimension $m$, and $\mathcal{N} \hookrightarrow \mathbb{R}^{N}$, then we define the $p$-energy of $u$ as

$$
\mathcal{E}_{p}(u) \doteq \int_{\mathcal{M}}|\nabla u(x)|_{\mathcal{N}}^{p} \omega_{g},
$$

where $\omega_{g}$ is the volume form associated to the metric $g$. Recall that, in a local coordinate chart, we have:

$$
|\nabla u(x)|=\sqrt{g^{i j}(x)\left\langle\frac{\partial u}{\partial x_{i}}(x), \frac{\partial u}{\partial x_{j}}(x)\right\rangle_{\mathcal{N}}}
$$




$$
\omega_{g}=\sqrt{\operatorname{det} g} d x_{1} \cdots d x_{m}
$$

where the scalar product $\langle\cdot, \cdot\rangle_{\mathcal{N}}$ is the Riemannian scalar product in the target $\mathcal{N}$.

Definition 1.2 (Singular set of a map) If $u \in W^{1, p}(\mathcal{M}, \mathcal{N})$, we define its singular set as

$$
\mathcal{S}(u) \doteq\{x \in \mathcal{M} \mid u \text { is not continuous at } x\}
$$

Assumptions on the domain. Since the problem of studying $\mathcal{S}(u)$ can be treated as a local problem, from now on we assume the setting to be the following: $u$ is defined on an open domain $\Omega$ of $\mathbb{R}^{m} ; \Omega$ contains a ball $B_{\bar{R}}(0)$ of a suitable radius $\bar{R}>2$; and we study the intersection $\mathcal{S}(u) \cap B_{1}(0)$. We won't be more precise than this about the actual size we need for $B_{\bar{R}}(0)$ : this will be the result of a collection of assumptions we will gradually make in the next sections. Notice that, given this assumption, the $p$ energy $\mathcal{E}_{p}(u)$ actually assumes the simpler form

$$
\mathcal{E}_{p}(u)=\int_{\Omega}\left(\sum_{i=1}^{m} \sum_{\alpha=1}^{N}\left(\frac{\partial u^{\alpha}}{\partial x_{i}}(x)\right)^{2}\right)^{\frac{p}{2}} d x
$$

for any map $u \in W^{1, p}(\Omega, \mathcal{N})$.

Remark When the domain is a generic smooth Riemannian manifold $(\mathcal{M}, g)$ of dimension $m$, the problem can be reduced to the Euclidean setting by suitably choosing a coordinate chart around a given point (see [6, Section 7]). For any point $q \in \mathcal{M}$, a system of normal coordinates is defined on a geodesic ball $B_{\varepsilon}^{\mathcal{M}}(q)$ around $q$; in these coordinates, the metric $g$ is represented by $g_{i j}(q)=\delta_{i j}$ at the point $q$. By smoothness, up to lowering the radius $\varepsilon$, we can assume that $|g-\mathrm{Id}|$ is small in $B_{\varepsilon}^{\mathcal{M}}(q)$. Under this assumption, one can give lower and upper bounds on the $p$-energy

$$
\mathcal{E}_{p, g}(u)=\int_{B_{\mathcal{\varepsilon}}^{\mathcal{M}}(q)}|\nabla u|_{g}^{p} \sqrt{\operatorname{det} g} d x
$$

in terms of

$$
\mathcal{E}_{p, \text { flat }}\left(u \circ \phi^{-1}\right)=\int_{B_{\varepsilon}(0)}\left|\nabla\left(u \circ \phi^{-1}\right)\right|^{p} d x,
$$

where $\phi: B_{\varepsilon}^{\mathcal{M}}(q) \rightarrow B_{\varepsilon}(0) \subset \mathbb{R}^{m}$ is the coordinate chart. Through this procedure, one can show that suitable versions of the main results we use ( $\varepsilon$-regularity, monotonicity formula) still hold (locally) in the case of non-flat manifolds (details and explicit computations can be found in [6, Section 7] and [21, Section 2.2]). Notice that, in this more general case, the quantitative results we prove will have constants depending on the injectivity radius of $\mathcal{M}$, due to the fact that we needed to choose normal coordinates on a geodesic ball. 
We are interested in studying minimizers of the $p$-energy, i.e. maps which minimize the functional $\mathcal{E}_{p}$ among those with the same boundary datum. More precisely, we give the following definitions:

Definition 1.3 Let $u \in W^{1, p}(\Omega, \mathcal{N})$. We say that:

1. $u$ is a $p$-energy minimizing map if $\mathcal{E}_{p}(u) \leq \mathcal{E}_{p}(v)$ for any compact set $K \subset \Omega$ and for any $v \in W^{1, p}(\Omega, \mathcal{N})$ such that $\left.\left.u\right|_{\Omega \backslash K} \equiv v\right|_{\Omega \backslash K}$ (more precisely: $u=v$ almost everywhere in $\Omega \backslash K$ ).

2. $u$ is a weakly $p$-harmonic map if it is a critical point of the $p$-energy functional with respect to variations in the target manifold, i.e.: for any $\xi \in \mathcal{C}_{c}^{\infty}\left(\Omega, \mathbb{R}^{N}\right)$, it holds:

$$
\left.\frac{d}{d t} \int_{\Omega}\left|\nabla\left(\Pi_{\mathcal{N}}(u+t \xi)\right)\right|^{p}\right|_{t=0}=0
$$

where $\Pi_{\mathcal{N}}$ is the nearest-point projection onto $\mathcal{N}$, defined on a tubular neighborhood of $\mathcal{N}$ itself.

3. $u$ is a stationary $p$-harmonic map if it is weakly $p$-harmonic and it is a critical point of the $p$-energy functional with respect to compact variations in the domain. Explicitly: let $\Phi=\left\{\phi_{t}\right\}_{t \in I}$ be any smooth family of diffeomorphisms of $\Omega$, with $I$ open interval containing 0 ; assume that $\phi_{0} \equiv \mathrm{id}_{\Omega}$, and that there exists a compact set $K \subset \Omega$ such that $\left.\phi_{t}\right|_{\Omega \backslash K}=\operatorname{id}_{\Omega \backslash K}$ for any $t \in I$; then

$$
\left.\frac{d}{d t} \mathcal{E}_{p}\left(u \circ \phi_{t}\right)\right|_{t=0}=\left.\frac{d}{d t} \int_{\Omega}\left|\nabla\left(u \circ \phi_{t}\right)(x)\right|^{p} d x\right|_{t=0}=0 .
$$

It is clear that any $p$-energy minimizing map is stationary $p$-harmonic. The results we achieve in this article will always be proved for $p$-energy minimizers. We now briefly survey the standard and classical tools available when dealing with $p$-minimizers: these techniques will be then expanded in Sects. 2 and 3.

\subsection{Basic Tools and References: An Overview}

Some essential references to keep in mind will be the work of Schoen and Uhlenbeck [19], for fundamental results regarding 2-harmonic maps, and its generalization to generic $p$, due to Hardt and Lin [6].

Monotonicity formula. Given a map $u \in W^{1, p}(\Omega, \mathcal{N})$ and a ball $B_{r}(x) \subset \Omega$, in Definition 2.3 we define a quantity $\theta(x, r)$ which will turn out to be scale invariant and monotone in $r$. This takes the same form of the normalized energy introduced in [19] for 2-harmonic maps and in [6] for $p$-harmonic maps (see also [18, Section 2.4]): indeed, we will consider the function

$$
\theta(x, r)=r^{p-m} \int_{B_{r}(x)}|\nabla u|^{p} d x .
$$


In Theorem 2.6 we will show that an explicit formula for $\frac{d}{d r} \theta(x, r)$ is available. As a consequence, it will be clear that $\theta(x, \cdot)$ is non-decreasing, and that $\theta(x, r)=\theta(x, s)$ with $r>s$ if and only if $u$ is 0-homogeneous in a ball of controlled radius centered at $x$. Making this statement quantitative will be a key idea:

Heuristic Principle: if the difference $\theta(x, r)-\theta(x, s)$ is small enough, then $u$ will be close to be 0-homogeneous in $B_{c r}(x)$; if this happens at $k+1$ points sufficiently far away from each other, then $u$ will be close to be invariant along a k-plane in a ball $B_{c r}\left(x_{0}\right)$.

We will turn this principle into a precise statement in Corollary 3.6; other version of the same idea will be used throughout the article.

Quantitative stratification. Based on ideas contained in [19], the following notion of singular stratification for a $W^{1, p}(\Omega, \mathcal{N})$ map can be introduced:

$$
\mathcal{S}^{k}(u) \doteq\{x \in \Omega \mid \text { any tangent map to } u \text { at } x \text { is at most } k \text {-symmetric }\} \text {; }
$$

here a tangent map is an $L^{p}$ limit of blow ups (which always exists thanks to the above monotonicity formula), and $k$-symmetric means 0 -homogeneous and invariant along a $k$-plane. For $p=2$, it was proved in [19] that $\operatorname{dim}_{\mathscr{H}} \mathcal{S}^{k}(u) \leq k$. In Definition 3.1 we give a quantitative version of this definition, based on the ones appearing in [2] for $p=2$ and [15] for $p$ generic: given three parameters $k, \eta$ and $r$ we look at points $x$ such that "in $B_{r}(x), u$ is not $\eta$-close to be $(k+1)$-invariant". We will say that any such point belongs to the stratum $\mathcal{S}_{\eta, r}^{k}(u)$. Another way to read the Heuristic Principle is that: if $x_{0} \in \mathcal{S}_{\eta, r}^{k}(u)$, then the points in $B_{c r}\left(x_{0}\right)$ for which $\theta(x, r)-\theta(x, s)$ is small must lie close to a k-plane.

$\varepsilon$-regularity. The link between the study of singular sets and the study of singular stratifications is an $\varepsilon$-regularity result (again from [6,19]): if $\theta(x, r)$ is sufficiently small, then $u$ is regular in $B \frac{r}{2}(x)$. With a simple compactness argument, one can generalize this result to prove that for some $\eta$ we have $\mathcal{S}(u) \subset \mathcal{S}_{\eta, r}^{m-\lfloor p\rfloor-1}$ (u) for any $r$ (see Proposition 3.3): thus the study of $\mathcal{S}(u)$ reduces to the study of the $(m-\lfloor p\rfloor-1)^{\text {th }}$ singular stratum.

\section{Preliminaries}

As in the case $p=2, p$-harmonic maps satisfy (in a weak sense) some suitable EulerLagrange equations, which are stated in the next two theorems (see for example [11, Sections 3.1 to 3.3] for a complete treatment of the case $p=2$; the same computations work for the case $p \in(1, \infty))$.

Theorem 2.1 (External variations) If u is weakly p-harmonic, then it satisfies (weakly) the equation

$$
\Delta_{p} u \doteq \operatorname{div}\left(|\nabla u|^{p-2} \nabla u\right)=-|\nabla u|^{p-2} A(u)(\nabla u, \nabla u),
$$


where $A$ is the second fundamental form of the embedding $\mathcal{N} \hookrightarrow \mathbb{R}^{N}$. Explicitly, we have

$$
\int_{\Omega}|\nabla u|^{p-2}\langle\nabla u, \nabla \phi\rangle d x=-\int_{\Omega}|\nabla u|^{p-2} A(u)(\nabla u, \nabla u) \phi d x,
$$

for any $\phi \in \mathcal{C}_{c}^{\infty}\left(\Omega, \mathbb{R}^{N}\right)$.

Example It is easy to show that the projection on the unit sphere $u: B_{1}^{N}(0) \rightarrow \mathbb{S}^{N-1}$ defined by $u(x)=\frac{x}{|x|}$ is in $W^{1, p}\left(B_{1}^{N}(0), \mathbb{S}^{N-1}\right)$ whenever $p<N$, and in that case it satisfies Eq. (ExtEL) classically out of the origin; as a consequence, one can immediately show that $u$ is weakly $p$-harmonic in $B_{1}^{N}(0)$. A key fact is that the second fundamental form of the sphere can be explicitly computed:

$$
A(x)(X(x), Y(x))=\langle X(x), Y(x)\rangle x \quad \forall x \in \mathbb{S}^{N-1} \text {. }
$$

It is then an easy exercise to show that the equation actually holds; both sides of the equation turn out to be equal to $-(N-1)^{\frac{p}{2}}|x|^{-1-p} x$.

Theorem 2.2 (Internal variations) If $u$ is stationary $p$-harmonic, then for any $X \in$ $\mathcal{C}_{c}^{\infty}\left(\Omega, \mathbb{R}^{m}\right)$

$$
\int_{\Omega}|\nabla u|^{p-2} \sum_{i, k=1}^{m}\left[p\left\langle\nabla_{i} u, \nabla_{k} u\right\rangle-|\nabla u|^{2} \delta_{i k}\right] \frac{\partial X^{k}}{\partial x^{i}} d x=0 .
$$

A crucial tool in the study of ( $p$-)harmonic maps is the normalized energy of a map in a fixed ball of the domain.

Definition 2.3 (Normalized energy) Let $u$ be a $W^{1, p}(\Omega, \mathcal{N})$ map. Let $\psi \in$ $\mathcal{C}_{c}^{\infty}([0, \infty))$ be a non-increasing function supported in $[0, \bar{R})$. For all $x \in B_{1}(0)$ and $r>0$ small enough, we define the normalized $p$-energy as the function

$$
\theta(x, r)=\theta_{\psi}[u](x, r) \doteq r^{p-m} \int_{\Omega} \psi\left(\frac{|y-x|}{r}\right)|\nabla u(y)|^{p} d y .
$$

The definition we give here is actually not the standard one (although it has already been used in several articles on the argument): one recovers the usual definition (present for example in $[2,13,19]$ ) by taking $\psi=\chi_{[0,1]}$ (which is not an admissible choice in our definition).

Remark (Motivation for the definition) As we will see in Theorem 2.6 (and all the subsequent results), a condition of the type $\theta_{\psi}(x, r)-\theta_{\psi}(x, s)=0$ with $r>s$ gives much more information on $u$ with our definition, rather than with the standard one: for example, one can only deduce that $u$ is 0-homogeneous in the annulus $\{y: s<$ $|y-x|<r\}$ if $\psi=\chi_{[0,1]}$, while we obtain 0-homogeneity in a whole ball around $x$ if we choose $\psi$ such that $\psi^{\prime}<0$ in an interval $(0, \bar{t})$. 
A first useful property of the normalized energy is that it is scale invariant:

Definition 2.4 (Blow-ups) Let $u: \Omega \rightarrow \mathcal{N}$, and let $B_{r}(x) \subset \Omega$. We define the blow-up of $u$ (centered at $x$, with scale $r$ ) as the map

$$
T_{x, r} u(y) \doteq u(x+r y)
$$

the definition makes sense on the set

$$
\frac{\Omega-x}{r} \doteq\left\{y \in \mathbb{R}^{m} \mid x+r y \in \Omega\right\} \supset B_{1}(0) .
$$

Theorem 2.5 (Scale invariance) If $u \in W^{1, p}(\Omega, \mathcal{N}), x \in B_{1}(0)$ and $r>0$, the following identity holds:

$$
\theta_{\psi}\left[T_{x, r} u\right](0,1)=\theta_{\psi}[u](x, r)
$$

As a further consequence, if also $w \in \frac{\Omega-x}{r}$ and $\tau>0$ is small enough, then:

$$
\theta_{\psi}\left[T_{x, r} u\right](w, \tau)=\theta_{\psi}[u](x+r w, r \tau) .
$$

Theorem 2.6 (Monotonicity formula) Let $u$ be a stationary $p$-harmonic map, and $\psi$ a smooth function as before. Fix $x \in B_{1}(0)$ and $r>0$ smaller than $\operatorname{dist}(x, \partial \Omega)$. Then $\theta_{\psi}(x, \cdot)$ has a derivative at $r$ and the following equality holds:

$$
\frac{d}{d r} \theta_{\psi}(x, r)=-p r^{p-m-2} \int_{\Omega}|y-x| \psi^{\prime}\left(\frac{|y-x|}{r}\right)|\nabla u(y)|^{p-2}\left|\partial_{r_{x}(y)} u(y)\right|^{2} d y,
$$

where for any $y$ we define $r_{x}(y) \doteq \frac{y-x}{|y-x|}$ to be the unit vector in the direction connecting $x$ to $y$.

Remark As we already mentioned in the remark at page 4, this statement is not true if the domain has a non-zero curvature: however, up to transforming the domain as we did in the aforesaid remark, it is indeed true that $e^{C r} \theta(x, r)$ is monotone, with $C$ only depending on $m$ and $p$ (see [6, Section 7] and [21, Theorem 2.7]). As it is easily seen, this modification does not affect our computations.

Proof We will proceed in two steps.

Step 1 We first consider the case $x=0, r=1$; the general case will then follow by scale invariance. In particular, we have to prove the following identity:

$$
\left.\frac{d}{d r} \theta_{\psi}(0, r)\right|_{r=1}=-p \int_{\Omega}|y| \psi^{\prime}(|y|)|\nabla u(y)|^{p-2}\left|\partial_{\frac{y}{|y|}} u(y)\right|^{2} d y .
$$

The key idea is to find a suitable vector field to plug into the Euler-Lagrange equation (IntEL): thus, we consider the following one:

$$
Y(y)=\psi(|y|) y \in \mathcal{C}_{c}^{\infty}\left(B_{\bar{R}}(0), \mathbb{R}^{m}\right)
$$


A simple computation gives, for $1 \leq i, j \leq m$,

$$
\frac{\partial Y^{j}}{\partial y^{i}}=\psi^{\prime}(|y|) \frac{y_{i} y_{j}}{|y|}+\psi(|y|) \delta_{i j}
$$

Then, with this choice of $Y$, the integral appearing in Eq. (IntEL) reads:

$$
\int_{\Omega}|\nabla u|^{p-2}\left[p|y| \psi^{\prime}(|y|)\left|\partial_{\frac{y}{|y|}} u\right|^{2}-|y| \psi^{\prime}(|y|)|\nabla u|^{2}+(p-m) \psi(|y|)|\nabla u|^{2}\right] d y
$$

this follows by a straightforward computation, and by the fact that:

$$
\sum_{i, j=1}^{m}\left\langle y_{i} \nabla_{i} u, y_{j} \nabla_{j} u\right\rangle=|y|^{2}\left\langle\sum_{i=1}^{m} \frac{y_{i}}{|y|} \nabla_{i} u, \sum_{j=1}^{m} \frac{y_{j}}{|y|} \nabla_{j} u\right\rangle=|y|^{2}\left|\partial_{\frac{y}{|y|}} u(y)\right|^{2} .
$$

Now by Eq. (IntEL) the integral in (2.8) is zero; hence Eq. (2.7) follows easily, just by taking the derivative of $\theta_{\psi}(0, \cdot)$ at $r=1$ (and changing the order of integral and derivative):

$$
\begin{aligned}
\frac{d}{d r} \theta_{\psi}(0, r)= & (p-m) r^{p-m-1} \int_{\Omega} \psi\left(\frac{|y|}{r}\right)|\nabla u(y)|^{p} d y+ \\
& +r^{p-m} \int_{\Omega} \psi^{\prime}\left(\frac{|y|}{r}\right)\left(-\frac{|y|}{r^{2}}\right)|\nabla u|^{p} d y .
\end{aligned}
$$

Step 2 Consider now the general case: arbitrarily fix $x \in B_{1}(0)$ and $\bar{r}>0$. By scale invariance, we know that $\theta_{\psi}[u](x, r)=\theta_{\psi}\left[T_{x, r} u\right](0,1)$ for all $r$ in a neighborhood of $\bar{r}$. Hence in particular

$$
\left.\frac{d}{d r} \theta_{\psi}[u](x, r)\right|_{r=\bar{r}}=\left.\frac{d}{d r} \theta_{\psi}\left[T_{x, r} u\right](0,1)\right|_{r=\bar{r}}
$$

Notice that by Step 1 we have information about the quantity $\frac{d}{d s} \theta_{\psi}\left[T_{x, \bar{r}} u\right](0, s)$ at $s=1$, which is not directly the information we seek, but is really close. Indeed, a simple computation (which involves nothing more than the definition of $T_{x, r}$ ) shows that the two quantities are related by

$$
\left.\frac{d}{d s} \theta_{\psi}\left[T_{x, \bar{r}} u\right](0, s)\right|_{s=1}=\left.\bar{r} \frac{d}{d r} \theta_{\psi}\left[T_{x, r} u\right](0,1)\right|_{r=\bar{r}}
$$


Thus we have:

$$
\begin{aligned}
\frac{d}{d r} \theta_{\psi}(x, \bar{r}) & =\left.\frac{1}{\bar{r}} \frac{d}{d s} \theta_{\psi}\left[T_{x, \bar{r}} u\right](0, s)\right|_{s=1} \\
& =-\frac{p}{\bar{r}} \int_{B_{\bar{R}}(0)}|y| \psi^{\prime}(|y|)\left|\nabla T_{x, \bar{r}} u(y)\right|^{p-2}\left|\partial_{\frac{y}{|y|}} T_{x, \bar{r}} u(y)\right|^{2} d y \\
& =-p \bar{r}^{p-1} \int_{B_{\bar{R}}(0)}|y| \psi^{\prime}(|y|)|\nabla u(x+\bar{r} y)|^{p-2}\left|\partial_{\frac{y}{|y|}} u(x+\bar{r} y)\right|^{2} d y .
\end{aligned}
$$

By performing the change of variables $w=x+\bar{r} y$, we obtain exactly the desired result.

Corollary 2.7 Let $\psi$ be a smooth function as before; define:

$$
\Psi(t) \doteq \int_{0}^{t} \tau^{p-m} \psi^{\prime}(\tau) d \tau .
$$

Then, for any $p$-stationary map $u$, for any $x \in B_{1}(0)$ and $0<s<r<\operatorname{dist}(x, \partial \Omega)$, we have:

$$
\begin{aligned}
\theta_{\psi}(x, r)-\theta_{\psi}(x, s)= & p \int_{\Omega}\left(\Psi\left(\frac{|y-x|}{r}\right)\right. \\
& \left.-\Psi\left(\frac{|y-x|}{s}\right)\right)|y-x|^{m-p}|\nabla u|^{p-2}\left|\partial_{r_{x}} u\right|^{2} d y
\end{aligned}
$$

Definition 2.8 (Assumptions on $\psi$ ) From now on, we will think of $\psi \in \mathcal{C}_{c}^{\infty}([0, \infty)$ ) as a fixed non-negative function, satisfying

$$
\begin{aligned}
& \operatorname{supp}(\psi)=\left[0, t_{b}\right], \\
& \psi^{\prime}(t)<0 \quad \text { in }\left[0, t_{b}\right), \quad \psi^{\prime}(t) \leq-\xi \quad \text { in }\left[0, t_{a}\right)
\end{aligned}
$$

for some fixed numbers $0<t_{a}<t_{b}$ and $\xi>0$ (see Figure 1). Moreover, since this will be needed in Sect. 6, we will actually assume $2<t_{a}<t_{b}$ : this choice will be better explained in the remark at page 24. The function in Figure 1 can be thought as a valid one.

Such a choice of $\psi$ is mostly justified by computational reasons; it's worth noting, however, that enlarging the value of $t_{b}$ is heuristically equivalent to looking at smaller balls in the domain, thus exploiting again the local nature of the problem.

A straightforward consequence of the monotonicity formula is the following:

Corollary 2.9 Let $0<s<r$; let $u \in W^{1,2}(\Omega, \mathcal{N})$ be a stationary harmonic map, and $x \in B_{1}(0)$. Take $\psi \in \mathcal{C}_{c}^{\infty}([0, \infty))$ as in Definition 2.8. If

$$
\theta(x, r)-\theta(x, s)=0,
$$


Fig. 1 Example for $\psi$, with $t_{b}=4, t_{a}=3.5, \xi=0.1$

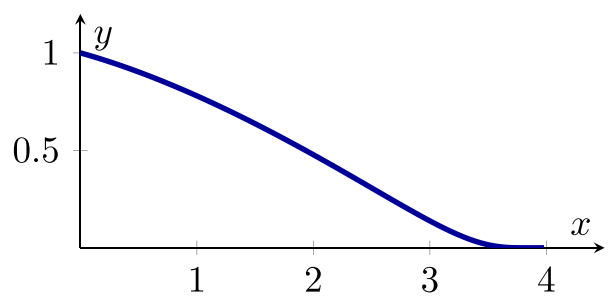

then $u$ is 0-homogeneous in $B_{t_{b} r}(x)$ (with respect to $x$ ); i.e., $u(y)=u(z)$ whenever $y, z \in B_{t_{b}}(x)$ and $y-x=\lambda(z-x)$ for some $\lambda>0$.

Moreover, by simple geometric considerations, if a map is 0-homogeneous with respect to different points, then it is invariant along the affine subspace generated by those points:

Corollary 2.10 (Rigidity) Let $0<s<r$; let $u \in W^{1, p}(\Omega, \mathcal{N})$ be a stationary harmonic map, and take $\psi \in \mathcal{C}_{c}^{\infty}([0, \infty))$ as in Definition 2.8. Let $0 \leq k \leq m$ be an integer. If there exist $k+1$ points $\left\{x_{i}\right\}_{i=0}^{k}$ such that:

- $x_{i} \in B_{\frac{1}{2} t_{b} r}\left(x_{0}\right) \subset \Omega$ for any $i=1, \ldots, k$;

- $\left\{x_{i}\right\}_{i=0}^{k}$ span a $k$-dimensional affine subspace $L$;

- For all $i=0, \ldots, k$,

$$
\theta\left(x_{i}, r\right)-\theta\left(x_{i}, s\right)=0
$$

then $u$ is 0-homogeneous at any point of $L \cap B_{\frac{1}{2} t_{b} r}\left(x_{0}\right)$ and $L$-invariant in $B_{\frac{1}{2} t_{b} r}\left(x_{0}\right)$, in the sense that $u(y)=u(z)$ whenever $y, z \in B_{\frac{1}{2} t_{b} r}\left(x_{0}\right)$ and $z-y \in L$.

\subsection{An $\epsilon$-Regularity Result}

Combining Theorems 2.5 and 3.1 of [6], we get the following Hölder-regularity result for $p$-minimizers:

Theorem 2.11 ( $\varepsilon$-regularity) There exist two constants $\varepsilon_{0}=\varepsilon_{0}(m, \mathcal{N}, \Lambda, p)$ and $\alpha(m, \mathcal{N}, \Lambda, p)$ such that the following holds. Let $u \in W^{1, p}(\Omega, \mathcal{N})$ be a minimizer for the p-energy, with $\mathcal{E}_{p}(u) \leq \Lambda$. If $\theta(x, r)<\varepsilon_{0}$ for some $B_{r}(x) \subset \Omega$, then $u$ is $\mathcal{C}^{1, \alpha}$-regular in $B_{\frac{r}{2}}(x)$.

This result will be the key argument that connects the singular set to the singular stratification (see Proposition 3.3).

Notice that the situation gets even better when dealing with 2-harmonic maps: by standard elliptic regularity arguments one can get $\mathcal{C}^{\infty}$ regularity instead of Hölder regularity (see $[17,19])$. 


\section{Quantitative Stratifications}

Let $u \in W^{1, p}(\Omega, \mathcal{N})$, and assume $L$ is a $k$-dimensional linear subspace of $\mathbb{R}^{m}$; we will use the symbol $\mathbf{G}^{k}\left(\mathbb{R}^{m}\right)$ for the collection of such subspaces. We denote by $\left|\nabla_{L} u\right|$ or $|\langle\nabla u, L\rangle|$ the quantity

$$
\left|\nabla_{L} u\right| \doteq\left(\sum_{i=1}^{k}\left|\left\langle\nabla u, v_{i}\right\rangle\right|^{2}\right)^{\frac{1}{2}}
$$

where $\left\{v_{1}, \ldots, v_{k}\right\}$ is any orthonormal basis of $L$.

Definition 3.1 (Quantitative stratification) Let $x \in B_{1}(0)$ and $r>0$ small enough for $B_{r}(x)$ to be contained in $\Omega$. Fix $k \in\{0, \ldots, m\}$ and a parameter $\eta>0$. We say that $u$ is $(\eta, k)$-invariant in $B_{r}(x)$ if there exists a linear subspace $L \in \mathbf{G}^{k}\left(\mathbb{R}^{m}\right)$ such that

$$
r^{p-m} \int_{B_{r}(x)}\left|\nabla_{L} u\right|^{p} d y<\eta .
$$

Equivalently, there exists $L \in \mathbf{G}^{k}\left(\mathbb{R}^{m}\right)$ such that

$$
\int_{B_{1}(0)}\left|\nabla_{L} T_{x, r} u\right|^{p}<\eta .
$$

When this condition holds for some $\eta$ and $k$, we will generically refer to it as "almost invariance". We define the singular $k^{\text {th }}$ stratum of $u$, with scale parameter $r$ and closeness parameter $\eta$ the subset of $B_{1}(0)$ defined by

$$
\begin{aligned}
\mathcal{S}_{\eta, r}^{k}(u) & \doteq\left\{x \in B_{1}(0) \mid u \text { is } \underline{\operatorname{not}}(\eta, k+1) \text {-invariant in } B_{s}(x) \text { for any } s \geq r\right\}= \\
& =\left\{\left.x \in B_{1}(0)\left|s^{p-m} \int_{B_{s}(x)}\right| \nabla_{L} u\right|^{p} d y \geq \eta \text { for all } L \in \mathbf{G}^{k+1}\left(\mathbb{R}^{m}\right) \text { and } s \geq r\right\} .
\end{aligned}
$$

Finally, we denote by $\mathcal{S}_{\eta}^{k}(u)$ the intersection

$$
\begin{aligned}
& \mathcal{S}_{\eta}^{k}(u) \doteq \bigcap_{r>0} \mathcal{S}_{\eta, r}^{k}(u) \\
& \quad=\left\{x \in B_{1}(0) \mid u \text { is } \underline{\operatorname{not}}(\eta, k+1) \text {-invariant in } B_{s}(x) \text { for any } s>0\right\},
\end{aligned}
$$

for any given $\eta$ and $k$.

Remark Notice that this definition is slightly different from the one used in [15] and originally introduced in [2]: indeed, in those papers $u$ is defined to be "almost $k$ invariant" if it is (quantitatively) close in $L^{p}$ to a map which is 0-homogeneous and $k$-invariant. The two approaches can be shown to be equivalent (see for example [7, Proposition 3.11]); we won't explore this path further. 
In the following proposition we show that the definition of almost invariance given above implies an "almost 0-homogeneity" condition at a smaller scale (and this is why we didn't require such a condition in the very definition of almost invariance). Not only: given a ball $B_{r}(x)$ where $u$ is $(\delta, k)$-invariant for a suitable $\delta$, all the points in $B_{\frac{1}{2} r}(x)$ are both almost invariant and almost 0-homogeneous with respect to this smaller scale.

Proposition 3.2 Let $\eta>0$ be a fixed parameter. There exists a constant $\bar{\gamma}(m, \mathcal{N}$, $\Lambda, p, \eta)$, with $0<\bar{\gamma}<\frac{1}{2}$, such that the following holds. Define $\bar{\delta} \doteq \bar{\gamma}^{2(m-p)}$. Let $u$ be a p-minimizing map with $\mathcal{E}_{p}(u) \leq \Lambda$, and $B_{r}(x) \subset B_{1}(0)$. Assume $u$ is $(\bar{\delta}, k)$-invariant in $B_{r}(x)$ :

$$
r^{p-m} \int_{B_{r}(x)}\left|\nabla_{L} u\right|^{p} d z<\bar{\delta}
$$

for some $L \in \mathbf{G}^{k}\left(\mathbb{R}^{m}\right)$. Then for any $y \in B_{\frac{1}{2} r}(x)$ there exists a radius $\bar{\gamma} r \leq r_{y} \leq$ $\frac{1}{2} r$ such that $u$ satisfies the following almost invariance and almost 0-homogeneity conditions in $B_{r_{y}}(y)$ :

$$
\begin{aligned}
& r_{y}^{p-m} \int_{B_{r_{y}}(y)}\left|\nabla_{L} u\right|^{p} d z<\eta \\
& \theta\left(y, r_{y}\right)-\theta\left(y, \frac{1}{2} r_{y}\right)<\eta .
\end{aligned}
$$

Proof By scale invariance, it is sufficient to prove the statement for $x=0, r=1$. Choose $\bar{\gamma}$ so that

$$
\bar{\gamma}<\min \left\{2^{-\frac{\Lambda}{\eta}-1}, \eta^{\frac{1}{m-p}}, \frac{1}{2}\right\} .
$$

Consider a point $y \in B_{\frac{1}{2}}(0)$; assume, by contradiction, that for all $i$ such that $2^{-i} \geq \bar{\gamma}$ we have

$$
\theta\left(y, 2^{-i}\right)-\theta\left(y, 2^{-i-1}\right) \geq \eta .
$$

Then we should have:

$$
\Lambda>\theta\left(y, \frac{1}{2}\right) \geq \sum_{i=1}^{\left\lfloor\frac{\Lambda}{\eta}+1\right\rfloor}\left(\theta\left(y, 2^{-i}\right)-\theta\left(y, 2^{-i-1}\right)\right) \geq\left\lfloor\frac{\Lambda}{\eta}+1\right\rfloor \eta>\Lambda
$$

a contradiction. Thus for any $y \in B_{\frac{1}{2}}(0)$ we have a radius $r_{y} \in\left[\bar{\gamma}, \frac{1}{2}\right]$ for which (3.8) holds. Moreover, by (3.9) we also have:

$$
r_{y}^{p-m} \int_{B_{r_{y}}(y)}\left|\nabla_{L} u\right|^{p} d z \leq \bar{\gamma}^{p-m} \bar{\delta}=\bar{\gamma}^{m-p}<\eta,
$$


which concludes the proof.

The bridge between singular stratification and singular set of a $p$-harmonic map is given by the following proposition, which strongly relies on the $\varepsilon$-regularity Theorem 2.11. This result can be seen as a quantitative version of the following known fact ( $[6$, Theorem 4.5]): a $p$-minimizing map which is 0 -homogeneous and invariant along a $(m-\lfloor p\rfloor)$-linear subspace must be constant.

Proposition 3.3 (Singular set and stratification) Let $u \in W^{1, p}(\Omega, \mathcal{N})$ be a p-energy minimizing map with energy bounded by $\Lambda$. There exists $\eta=\eta(m, \mathcal{N}, \Lambda, p)$ such that for all $r>0$ (small) we have

$$
\mathcal{S}(u) \cap B_{1}(0) \subset \mathcal{S}_{\eta, r}^{m-\lfloor p\rfloor-1}(u)
$$

Proof For any $i \in \mathbb{N}$, let $\gamma_{i} \doteq \bar{\gamma}\left(m, \mathcal{N}, \Lambda, p, \frac{1}{i}\right)$ be the constant given by Proposition 3.2 when $\eta=\frac{1}{i}$, and let $\delta_{i} \doteq \gamma_{i}^{2(m-p)}$.

We argue by contradiction: assume that for all $i \in \mathbb{N}$ there exists a $p$-minimizing map $u_{i}$ with $\mathcal{E}_{p}\left(u_{i}\right) \leq \Lambda$, a singular point $x_{i} \in \mathcal{S}\left(u_{i}\right)$, a $r_{i}>0$ and a $(m-\lfloor p\rfloor)$-plane $L_{i}$ such that

$$
\int_{B_{1}(0)}\left|\nabla_{L_{i}} T_{x_{i}, r_{i}} u_{i}\right|^{p}<\delta_{i}
$$

Up to precomposing with a rotation of the space, we can assume $L_{i}=L$ for all $i$, for some affine subspace $L$. By Proposition 3.2, we have

$$
\begin{aligned}
& \left(\alpha_{i} r_{i}\right)^{p-m} \int_{B_{\alpha_{i} r_{i}}\left(x_{i}\right)}\left|\nabla_{L} u_{i}\right|^{p} d z<\frac{1}{i} \\
& \theta\left[u_{i}\right]\left(x_{i}, \alpha_{i} r_{i}\right)-\theta\left[u_{i}\right]\left(x_{i}, \frac{1}{2} \alpha_{i} r_{i}\right)<\frac{1}{i}
\end{aligned}
$$

for a sequence $\left\{\alpha_{i}\right\}_{i}$, with $\gamma_{i} \leq \alpha_{i} \leq \frac{1}{2}$. The maps $T_{x_{i}, \alpha_{i} r_{i}} u_{i}$ are $p$-minimizing, and they are uniformly bounded in $W^{1, p}\left(B_{1+\varepsilon}(0), \mathcal{N}\right)$ for some $\varepsilon$ (by compactness of $\mathcal{N}$ and by the bound on the $p$-energy); thus, up to subsequences, they weakly converge to a map $\tilde{u} \in W^{1, p}\left(B_{1+\varepsilon}(0), \mathcal{N}\right)$. By [6,9], the convergence is actually strong in $W^{1, p}\left(B_{1}(0), \mathcal{N}\right)$, and $\tilde{u}$ is a $p$-minimizer in $B_{1}(0)$. But now by strong $W^{1, p}\left(B_{1}(0), \mathcal{N}\right)$ convergence and by (3.15), (3.16) we have

$$
\begin{aligned}
& \int_{B_{1}(0)}\left|\nabla_{L} \tilde{u}\right|^{p}=0, \\
& \theta[\tilde{u}](0,1)-\theta[\tilde{u}]\left(0, \frac{1}{2}\right)=0
\end{aligned}
$$

so $\tilde{u}$ is $p$-minimizing, $(m-\lfloor p\rfloor)$-invariant on $B_{1}(0)$ and 0 -homogeneous on $B_{1}(0)$. By $\left[6\right.$, Theorem 4.5], this implies that $\tilde{u}$ is constant on $B_{1}(0)$ : thus in particular $\theta[\tilde{u}](0, \cdot) \equiv$ 
0 in $(0,1)$. However, by the fact that $x_{i} \in \mathcal{S}\left(u_{i}\right)$, and by the $\varepsilon$-regularity Theorem 2.11 , we have:

$$
\theta\left[T_{x_{i}, \alpha_{i} r_{i}} u_{i}\right](0, s) \geq \varepsilon_{0} \quad \forall s>0,
$$

which implies $\theta[\tilde{u}](0, s) \geq \varepsilon_{0}$ by $W^{1, p}$-convergence: we have reached a contradiction.

Since we will make great use of compactness and limiting arguments, we will need an effective notion of "points in general position" which is preserved when passing to the limit. We will denote by $\mathbf{H}^{k}\left(\mathbb{R}^{m}\right)$ the space of affine $k$-dimensional subspaces of $\mathbb{R}^{m}$.

Definition 3.4 Given $k+1$ points $\left\{x_{i}\right\}_{i=0}^{k}$ in $\mathbb{R}^{m}$ (with $0 \leq k \leq m$ ), and $\varrho>0$, we say that $\left\{x_{i}\right\}_{i}$ are in $\varrho$-general position if for all $j=1, \ldots, k$

$$
\operatorname{dist}\left(x_{j}, x_{0}+\operatorname{span}\left\{x_{1}-x_{0}, \ldots, x_{j-1}-x_{0}\right\}\right) \geq \varrho .
$$

We say that a set of points $\mathcal{S}$ spans $\varrho$-effectively a given $k$-subspace $L \in \mathbf{H}^{k}\left(\mathbb{R}^{m}\right)$ if there exist $k+1$ points $\left\{x_{0}, x_{1}, \ldots, x_{k}\right\} \subset \mathcal{S}$ in $\varrho$-general position such that

$$
L=x_{0}+\operatorname{span}\left\{x_{1}-x_{0}, \ldots, x_{k}-x_{0}\right\}
$$

As we wanted, the notion of $\varrho$-general position passes to the limit:

Lemma 3.5 For any $j \in \mathbb{N}$, let $\left\{x_{i j}\right\}_{i=0}^{k}$ be $k+1$ points of $\mathbb{R}^{m}$ in $\varrho$-general position, with $\varrho>0$. Assume that $x_{i j} \stackrel{j \rightarrow \infty}{\longrightarrow} \bar{x}_{i}$ for all $i=0, \ldots, k$. Then $\left\{\bar{x}_{i}\right\}_{i=0}^{k}$ are still in Q-general position.

We are now ready to state a first precise version of what we called "Heuristic Principle" in Sect. 1.2. This makes Corollary 2.10 quantitative; the proof is a simple compactness argument, based on Lemma 3.5 and on the (already used) fact that weak $W^{1, p}$ limits of $p$-minimizing maps are actually strong limits, and are $p$-minimizers themselves.

Corollary 3.6 (Quantitative rigidity) Let $u \in W^{1, p}(\Omega, \mathcal{N})$ be a p-minimizing map with energy bounded by $\Lambda$. Let $0 \leq k \leq m$ be an integer. Fix the constants $\eta, p, \gamma, \varrho>0$. There exists a constant $\varepsilon>0$ (depending on $m, \mathcal{N}, \Lambda, \eta, p, \gamma, \varrho$ ) suich that the following holds. If there exist $k+1$ points $\left\{x_{i}\right\}_{i=0}^{k}$ such that:

- $x_{i} \in B_{\frac{1}{2} t_{b} r}\left(x_{0}\right) \subset \Omega$ for any $i=1, \ldots, k$;

- $\left\{x_{i}\right\}_{i=0}^{k}$ span $\varrho$-effectively a $k$-dimensional affine subspace $L$;

- For all $i=0, \ldots, k$,

$$
\theta\left(x_{i}, r\right)-\theta\left(x_{i}, \gamma r\right)<\varepsilon
$$

then $r^{p-m} \int_{B_{t_{b}}\left(x_{0}\right)}\left|\nabla_{L} u\right|^{p}<\eta$. 


\subsection{The Results: Precise Statements}

The main result we will prove is the following.

Theorem 3.7 (Singular strata) Let $u \in W^{1, p}(\Omega, \mathcal{N})$ be a p-energy minimizing map with energy bounded by $\Lambda$. Let $\eta>0$ and $1 \leq k \leq m$. There exists two constants $C_{1}$ and $\delta_{0}$ depending on $m, \mathcal{N}, p, \Lambda, \eta$ such that for any $r>0$

$$
\operatorname{Vol}\left(\mathcal{B}_{r}\left(\mathcal{S}_{\eta, \delta_{0} r}^{k}(u)\right) \cap B_{1}(0)\right) \leq C_{1} r^{m-k} .
$$

Moreover, for any $\eta>0$ and any $0 \leq k \leq m$, the stratum $\mathcal{S}_{\eta}^{k}(u)$ is $k$-rectifiable.

The proof will be achieved in Sect. 7, exploiting all the tools developed in Sects. 4 to 6.

Notice that, thanks to Proposition 3.3, we obtain the following crucial corollary, which is actually the main goal we wanted to achieve: volume estimates and structural information for the singular set.

Corollary 3.8 (Singular set) Let $u \in W^{1, p}(\Omega, \mathcal{N})$ be a p-energy minimizing map with energy bounded by $\Lambda$. There exists a constant $C_{2}(m, \mathcal{N}, \Lambda, p)$ such that for any $r>0$

$$
\operatorname{Vol}\left(\mathcal{B}_{r}(\mathcal{S}(u)) \cap B_{1}(0)\right) \leq C_{2} r^{\lfloor p\rfloor+1} .
$$

In particular, the Minkowski (and Hausdorff) dimension of $\mathcal{S}(u)$ is at most $m-\lfloor p\rfloor-1$, and the upper Minkowski content is bounded by $C_{2}$. Furthermore, $\mathcal{S}(u)$ is $(m-\lfloor p\rfloor-$ 1)-rectifiable.

\section{Reifenberg Theorems and Approximating Planes}

The next step will be to introduce some more advanced techniques which allow us to analyze the behaviour of each singular stratum at every scale $r$ around a point $x$. In order to state Reifenberg Theorem (in a form which is suited to our context), we first need to recall the definition of Jones' numbers of a measure $\mu$ (first appeared in [8]; for a detailed introduction, see [16]): this is a scale-invariant notion which quantifies how close $\operatorname{supp}(\mu)$ is to be contained in an affine $k$-space (near a given point).

Definition 4.1 [Jones' numbers] Let $x \in B_{1}(0)$ and $0<r<1$. Assume $\mu$ is a positive Radon measure on $\Omega$. For any $k \in\{0, \ldots, m\}$ we define the $k$-dimensional Jones' number of $\mu$ in $B_{r}(x)$ as

$$
\beta_{\mu}^{k}(x, r) \doteq\left(r^{-k} \inf \left\{\int_{B_{r}(x)} \frac{\operatorname{dist}(y, L)^{2}}{r^{2}} d \mu(y) \mid L \in \mathbf{H}^{k}\left(\mathbb{R}^{m}\right)\right\}\right)^{\frac{1}{2}}
$$

Remark The quantity $\beta_{\mu}^{k}$ is scale invariant in the following sense. Assume $\mu$ is a measure on a ball $B_{r}(x)$; define the blow up measure $\hat{\mu}=\hat{\mu}_{x, r}^{k}$ on $B_{1}(0)$ as

$$
\hat{\mu}(A) \doteq r^{-k} \mu(x+r A) \quad \forall A \subset B_{1}(0) \text { measurable. }
$$


Then it is easy to compute that $\beta_{\hat{\mu}}^{k}(0,1)=\beta_{\mu}^{k}(x, r)$.

The main hypothesis one needs, in order to apply Reifenberg Theorem in its different forms, is a control on the Jones numbers of a suitable measure (e.g., $\mathscr{H}^{k}$ restricted to a set). In all the cases, the condition we need takes the following form:

Definition 4.2 (Reifenberg condition) Let $\mu$ be a positive Radon measure on $\Omega$, and $k \in\{0, \ldots, m\}$. We say that $\mu$ satisfies the ( $k$-dimensional) Reifenberg condition with constant $\delta$ if for any $x \in B_{1}(0)$ and $0<r<1$ we have:

$$
\int_{B_{r}(x)} \int_{0}^{r} \beta_{\mu}^{k}(y, s)^{2} \frac{d s}{s} d \mu(y)<\delta r^{k} .
$$

As we will clarify in Theorem 4.4, two versions of Reifenberg Theorem are available for our purposes: in one of them (necessary for the rectifiability of a set), one needs to check ( $k$-Reif) on the restriction of the Hausdorff measure to the given set; the other one (necessary for volume estimates) makes use of discrete measures as the following:

Definition 4.3 (Measure associated to a disjoint family of balls) Assume $\mathcal{C}$ is a (discrete) subset of $B_{1}(0)$, and $\mathscr{F}=\left\{B_{r_{x}}(x)\right\}_{x \in \mathcal{C}}$ is a collection of disjoint balls centered in $\mathcal{C}$, each contained in $B_{2}(0)$. For any $k \in\{0, \ldots, m\}$, we define the following measure associated to $\mathscr{F}$ :

$$
\mu_{\mathscr{F}, k} \doteq \sum_{x \in \mathcal{C}} r_{x}^{k} \delta_{x}
$$

where $\delta_{x}$ is the Dirac measure centered at $x$.

Theorem 4.4 (Reifenberg) There exist two constants $C_{R}$ and $\delta_{R}$ such that the following statements hold true.

(i) Assume $\mathscr{F}$ is a family of disjoint balls with centers in $\mathcal{C} \subset B_{1}(0)$, each contained in $B_{2}(0)$. If $\mu_{\mathscr{F}, k}$ satisfies the condition ( $k$-Reif) with constant $\delta_{R}$, then

$$
\sum_{x \in \mathcal{C}} r_{x}^{k} \leq C_{R}
$$

(ii) Assume $S \subset B_{1}(0)$ is a $\mathscr{H}^{k}$-measurable set. If $\mathscr{H}^{k}\llcorner S$ satisfies the condition ( $k$-Reif) with constant $\delta_{R}$, then $S$ is $k$-rectifiable and

$$
\mathscr{H}^{k}\left\llcorner S\left(B_{r}(x)\right) \leq C_{R} r^{k}\right.
$$

for any $x \in S$ and $0<r<1$.

The original proof of this version of Reifenberg Theorem can be found in [13, Sections 5 and 6]; a more general form of it is contained in [4, Section 2], while similar arguments are developed in $[1,3,5,10,20]$.

Since we will need it in this form, for the sake of clarity we state here a rescaled version of Theorem 4.4, part (i). 
Corollary 4.5 (Reifenberg, rescaled version) Let $B_{\bar{r}}(\bar{x})$ be a fixed ball. Assume $\mathscr{F}$ is a family of disjoint balls with centers in $\mathcal{C} \subset B_{\bar{r}}(\bar{x})$, each contained in $B_{2 \bar{r}}(\bar{x})$. If $\mu=\sum_{x \in \mathcal{C}} r_{x}^{k} \delta_{x}$ satisfies

$$
\int_{B_{\bar{r} r}(w)} \int_{0}^{\bar{r} r} \beta_{\mu}^{k}(y, s)^{2} \frac{d s}{s} d \mu(y)<\delta_{R}(\bar{r} r)^{k}
$$

for all $w \in B_{\bar{r}}(\bar{x})$ and all $0<r<1$, then

$$
\sum_{x \in \mathcal{C}} r_{x}^{k} \leq C_{R} \bar{r}^{k}
$$

Proof It suffices to apply Theorem 4.4 to the measure $\hat{\mu}_{\bar{x}, \bar{r}} \doteq \bar{r}^{-k} T_{\bar{x}, \bar{r}} \sharp \mu$ introduced in the remark above: by using the change of variable formula for the integral, and exploiting the scale invariance of $\beta^{k}$ we obtain the result.

Remark Notice that the constants $\delta_{R}$ and $C_{R}$ for the rescaled version are the same as in Theorem 4.4, and thus only depending on $m$.

\subsection{Estimates on Jones' Numbers}

The key estimate, linking the Jones' numbers of a measure with the normalized $p$ energy of a $p$-minimizing map, is given in the following theorem, which we prove in several steps.

Theorem 4.6 (Estimates on $\beta_{\mu}^{k}$ ) Let $u \in W^{1, p}(\Omega, \mathcal{N})$ be a p-energy minimizing map. Fix the following constants: $0<\bar{r} \leq 1, \eta>0, \sigma>1, k \in\{1, \ldots, m\}$. Let $x \in B_{1}(0)$ and $r>0$. Assume $u$ is $\underline{\text { not }}(\eta, k+1)$-invariant in $B_{\bar{r} r}(x)$. There exists a constant $C_{3}(m, p, \eta, \sigma, \bar{r})$ such that the following estimate

$$
\beta_{\mu}^{k}(x, r)^{2} \leq C_{3} r^{-k} \int_{B_{r}(x)}(\theta(y, \sigma r)-\theta(y, r)) d \mu(y)
$$

holds for any positive Radon measure $\mu$ on $\Omega$.

Remark When this theorem will be used in Sect. 6, we will assign a precise value to $\sigma$; notice that in order that all the expressions involved are meaningful we could need to enlarge the domain $\Omega$ (and thus $\bar{R}$ ) according to $\sigma$ (see Assumptions on the domain at page 4$)$.

Remark By scale invariance (of both $\beta_{\mu}^{k}$ and $\theta$ ), it will be enough to prove the estimate for $x=0$ and $r=1$. Moreover, since the inequality does not change when multiplied by a constant, we can assume $\mu$ is a probability measure on $B_{1}(0)$. That is: assuming $u$ is not $(\eta, k+1)$-invariant in $B_{\bar{r}}(0)$, we will prove that

$$
\beta_{\mu}^{k}(0,1)^{2} \leq C_{3} \int_{B_{1}(0)}(\theta(y, \sigma)-\theta(y, 1)) d \mu(y)
$$


for any measure $\mu$ with $\mu\left(B_{1}(0)\right)=1$.

Summary of the proof. First of all, we find an explicit expression for the Jones' number of a measure. This relies on the fact that the infimum among affine planes which defines $\beta_{\mu}^{k}$ is actually achieved, and the minimal plane has a particularly manageable characterization (Subtheorem 4.6.1).

Secondly (and separately from the first point), we use directly the monotonicity formula (MF) to estimate the "radial $p$-energy" of $u$ in a ball with the quantity $\theta(\cdot, \sigma)-$ $\theta(\cdot, 1)$ computed at the center of the ball (Subtheorem 4.6.2).

Next, we estimate the $p$-energy of $u$ along some selected directions $v_{j} \in \mathbb{S}^{m-1}$, exploiting the information we got from the second step; here the explicit expression for $\beta_{\mu}^{k}$ emerges (Subtheorem 4.6.3).

Finally, we consider $k+1$ selected directions together: we give an upper bound to the $p$-energy of $u$ along a $(k+1)$-plane in terms of $\theta(\cdot, \sigma)-\theta(\cdot, 1)$. We then use the fact that $u$ is not $(k+1)$-almost invariant at 0 to say that the same $p$-energy must be at least some fixed amount.

We begin by writing the Jones' number in an explicit way. Some preliminary definitions are needed.

Definition 4.7 Let $\mu$ be a measure with support in $B_{1}(0)$. We define:

(i) the center of mass of $\mu$ as the point $x_{c m}^{\mu} \in B_{1}(0)$ such that

$$
x_{c m}^{\mu} \doteq f_{B_{1}(0)} x d \mu(x)
$$

(ii) the second moment of $\mu$ as the bilinear form $Q^{\mu}$ such that for all $v, w \in \mathbb{R}^{m}$

$$
Q^{\mu}(v, w) \doteq \int_{B_{1}(0)}\left[\left(x-x_{c m}\right) \cdot v\right]\left[\left(x-x_{c m}\right) \cdot w\right] d \mu(x) .
$$

We will usually drop the superscript $\mu$ when it is clear from the context.

Since $Q$ is symmetric and positive-definite, by the Spectral Theorem the associated matrix (which we still denote by $Q$ ) admits an orthonormal basis of eigenvectors, with non-negative eigenvalues. We denote with $\lambda_{1}^{\mu}, \ldots, \lambda_{m}^{\mu}$ the eigenvalues of $Q$ in decreasing order, and with $v_{1}^{\mu}, \ldots, v_{m}^{\mu}$ the respective eigenvectors (pairwise orthogonal and of norm 1), again dropping the superscripts when they are clear; in particular:

$$
\begin{aligned}
& \lambda_{k} v_{k}=\int_{B_{1}(0)}\left[\left(x-x_{c m}\right) \cdot v_{k}\right]\left(x-x_{c m}\right) d \mu(x) ; \\
& \lambda_{1} \geq \lambda_{2} \geq \cdots \geq \lambda_{m} .
\end{aligned}
$$

Finally, we denote by $V_{k}^{\mu}$ (or $V^{k}$ ) the following affine $k$-plane:

$$
\begin{aligned}
& V_{k}^{\mu} \doteq x_{c m}^{\mu}+W_{k}^{\mu} \\
& W_{k}^{\mu} \doteq \operatorname{span}\left\{v_{1}^{\mu}, \ldots, v_{k}^{\mu}\right\} .
\end{aligned}
$$


We are now ready to characterize $\beta_{\mu}^{k}$ :

Subtheorem 4.6.1 Let $\mu$ be a measure on $B_{1}(0)$. The affine space $V_{k}^{\mu}$ achieves the minimum in the definition of $\beta_{\mu}^{k}(0,1)$. Moreover,

$$
\beta_{\mu}^{k}(0,1)=\int_{B_{1}(0)} \operatorname{dist}^{2}\left(y, V_{k}^{\mu}\right) d \mu(y)=\lambda_{k+1}^{\mu}+\cdots+\lambda_{m}^{\mu}
$$

For a proof of this fact, see [13, Lemma 7.4] or [14, Subsection 6.1]. This is based on the (visually helpful) fact that the eigenvalues $\lambda_{k}$ and eigenvectors $v_{k}$ admit the following characterization:

- $\lambda_{1}$ is the maximum of $\int_{B_{1}(0)}\left\langle x-x_{c m}, v\right\rangle^{2} d \mu$ among vectors $v$ of norm 1 , and $v_{1}$ is any maximizing vector;

- $\lambda_{k}$ is the maximum of the same operator among all unit vectors orthogonal to $v_{1}, \ldots, v_{k-1}$, and $v_{k}$ is any maximizing vector.

We have thus reduced the problem to showing:

$$
\lambda_{k+1}^{\mu}+\cdots+\lambda_{m}^{\mu} \leq C \int_{B_{1}(0)}(\theta(y, \sigma)-\theta(y, 1)) d \mu(y)
$$

or, since the eigenvalues are in decreasing order, we need to show even less:

$$
\lambda_{k+1}^{\mu} \leq C \int_{B_{1}(0)}(\theta(y, \sigma)-\theta(y, 1)) d \mu(y) .
$$

The following result gives an estimate involving the difference $\theta(y, \sigma)-\theta(y, 1)$. It is really a direct consequence of the monotonicity formula (and our choice of $\psi$ ): none of the tools just introduced is required. Recall that $\xi$ and $t_{a}$ are the constants introduced in Definition 2.8.

Subtheorem 4.6.2 Let $u \in W^{1, p}(\Omega, \mathcal{N})$ be p-minimizing, $y \in B_{1}(0)$; assume $R_{1}, R_{2}$ and $R$ are radii satisfying $R_{2} \leq \frac{R}{t_{a}}<R_{1}$. Then the following inequality holds for some constant $C_{4}\left(m, R, R_{1}, p\right)$ (provided both sides are well defined):

$$
\int_{B_{R}(y)}|\nabla u(z)|^{p-2}|\langle\nabla u(z), y-z\rangle|^{2} d z \leq C_{4}\left(\theta\left(y, R_{1}\right)-\theta\left(y, R_{2}\right)\right) .
$$

In particular, taking $R_{2}=1, R=\max \left\{2, t_{a}\right\}$, and $R_{1}>R / t_{a}$ we also get

$$
\int_{B_{1}(0)}|\nabla u(z)|^{p-2}|\langle\nabla u(z), y-z\rangle|^{2} d z \leq C_{5}\left(\theta\left(y, R_{1}\right)-\theta(y, 1)\right),
$$

with $C_{5}$ depending only on $R_{1}, m, p$. 
Proof By Theorem 2.6:

$$
\begin{aligned}
& \theta\left(y, R_{1}\right)-\theta\left(y, R_{2}\right) \geq \int_{\frac{R}{t_{a}}}^{R_{1}} p r^{p-m-2} \\
& \int_{B_{R}(y)}|z-y|\left[-\psi^{\prime}\left(\frac{|z-y|}{r}\right)\right]|\nabla u|^{p-2}\left|\partial_{r_{y}} u\right|^{2} d z d r .
\end{aligned}
$$

Since $z \in B_{R}(y)$ and $r \geq \frac{R}{t_{a}}$, by the assumptions on $\psi$ made in Definition 2.8 we have:

$$
-\psi^{\prime}\left(\frac{|z-y|}{r}\right) \geq \xi
$$

The two integrals can be then separated and we get:

$$
\begin{gathered}
\theta\left(y, R_{1}\right)-\theta\left(y, R_{2}\right) \geq p \xi \frac{R_{1}^{p-m-1}-\left(\frac{R}{t_{a}}\right)^{p-m-1}}{p-m-1} \\
\int_{B_{R}(y)}|\nabla u(z)|^{p-2} \frac{|\langle\nabla u(z), z-y\rangle|^{2}}{|z-y|} d z .
\end{gathered}
$$

But now notice that $|z-y|^{-1} \geq R^{-1}$ : inequality (4.15) is proved. The last statement (Eq. (4.16)) follows from the fact that $B_{1}(0) \subset B_{R}(y)$, because $y \in B_{1}(0)$ and $R \geq 2$.

Next, we use the "radial" information we just achieved to estimate the $p$-energy along the eigenvectors $v_{j}$. Notice that the eigenvalues $\lambda_{j}$ also appear in the estimate.

Subtheorem 4.6.3 Let $u$ be p-minimizing, and $\mu$ a Radon measure on $B_{1}(0)$; let $\left\{\lambda_{j}\right\}_{j}$, $\left\{v_{j}\right\}_{j}$ be the eigenvalues and eigenvectors of $Q^{\mu}$, as before. There exists a constant $C_{6}(m, \mathcal{N}, p)$ such that for all $j=1, \ldots, m$ the following holds:

$$
\lambda_{j} \int_{B_{1}(0)}|\nabla u|^{p-2}\left|\left\langle\nabla u, v_{j}\right\rangle\right|^{2} d z \leq C_{6} \int_{B_{1}(0)}(\theta(y, \sigma)-\theta(y, 1)) d \mu(y) .
$$

Proof Up to performing a translation, we can assume $x_{c m}=0$. By definition of the eigenvalue $\lambda_{j}$, we have

$$
\lambda_{j} v_{j}=\int_{B_{1}(0)}\left\langle y, v_{j}\right\rangle y d \mu(y) .
$$

Fix $z \in B_{1}(0)$. By taking the scalar product of $\nabla u(z)$ with both sides of the previous equality, we obtain:

$$
\lambda_{j}\left\langle\nabla u(z), v_{j}\right\rangle=\int_{B_{1}(0)}\left\langle y, v_{j}\right\rangle\langle\nabla u(z), y\rangle d \mu(y)
$$

moreover, since $x_{c m}=0$, we have: 


$$
\int_{B_{1}(0)}\left\langle y, v_{j}\right\rangle\langle\nabla u(z), z\rangle d \mu(y)=\langle\nabla u(z), z\rangle\left\langle\int_{B_{1}(0)} y d \mu(y), v_{j}\right\rangle=0 .
$$

By subtracting (4.23) to (4.22), and multiplying both sides by $|\nabla u(z)|^{\frac{p}{2}-1}$, we can write:

$$
\lambda_{j}|\nabla u(z)|^{\frac{p}{2}-1}\left\langle\nabla u(z), v_{j}\right\rangle=\int_{B_{1}(0)}\left\langle y, v_{j}\right\rangle|\nabla u(z)|^{\frac{p}{2}-1}\langle\nabla u(z), y-z\rangle d \mu(y) .
$$

We then take the squared norms of both sides and apply Hölder inequality:

$$
\begin{aligned}
\lambda_{j}^{2}|\nabla u(z)|^{p-2}\left|\left\langle\nabla u(z), v_{j}\right\rangle\right|^{2} \leq & \int_{B_{1}(0)}\left\langle y, v_{j}\right\rangle^{2} d \mu(y) . \\
& \cdot \int_{B_{1}(0)}|\nabla u(z)|^{p-2}|\langle\nabla u(z), y-z\rangle|^{2} d \mu(y) \\
= & \lambda_{j} \int_{B_{1}(0)}|\nabla u(z)|^{p-2}|\langle\nabla u(z), y-z\rangle|^{2} d \mu(y) .
\end{aligned}
$$

Now if $\lambda_{j}=0$ the statement is trivial, so we can assume $\lambda_{j}>0$ (since all the eigenvalues are non-negative); we are thus allowed to divide by $\lambda_{j}$. Equation (4.25) holds for all $z \in B_{1}(0)$, thus we can then integrate both sides on $B_{1}(0)$ with respect to the Lebesgue measure in the variable $z$. At this point we get, also using Tonelli's Theorem:

$$
\begin{aligned}
& \lambda_{j} \int_{B_{1}(0)}|\nabla u(z)|^{p-2}\left\langle\nabla u(z), v_{j}\right\rangle^{2} d z \\
& \quad \leq \int_{B_{1}(0)} \int_{B_{1}(0)}|\nabla u(z)|^{p-2}|\langle\nabla u(z), y-z\rangle|^{2} d z d \mu(y) .
\end{aligned}
$$

Hence, a direct application of Subtheorem 4.6.2 gives the desired result.

Now, thanks to the last result, we have an upper bound on the $p$-energy along the $(k+1)$-plane $V_{k}$ introduced in Eq. (4.12); but this is bounded from below by a constant, by the lack of almost invariance in 0 . We have all the ingredients to complete the proof of Theorem 4.6.

Proof of Theorem 4.6 Applying Subtheorem 4.6.3 to $\lambda_{1}, \ldots, \lambda_{k+1}$, and recalling that the $\lambda_{j}$ 's are ordered decreasingly, we get:

$$
\begin{aligned}
\lambda_{k+1} \int_{B_{1}(0)}|\nabla u|^{p-2}\left|\left\langle\nabla u, V_{k+1}^{\mu}\right\rangle\right|^{2} d z & \leq \sum_{j=1}^{k+1} \lambda_{j} \int_{B_{1}(0)}|\nabla u|^{p-2}\left|\left\langle\nabla u, v_{j}\right\rangle\right|^{2} d z \\
& \leq(k+1) C_{6} \int_{B_{1}(0)}(\theta(y, \sigma)-\theta(y, 1)) d \mu(y) .
\end{aligned}
$$


On the other hand: since $u$ is not $(\eta, k+1)$-invariant in $B_{\bar{r}}(0)$, and $V_{k+1}^{\mu}$ is a $(k+1)$ plane, we have by definition:

$$
\begin{aligned}
\int_{B_{1}(0)}|\nabla u|^{p-2}\left|\left\langle\nabla u, V_{k+1}^{\mu}\right\rangle\right|^{2} d z & \geq \int_{B_{1}(0)}\left|\left\langle\nabla u, V_{k+1}^{\mu}\right\rangle\right|^{p} d z \\
& \geq \int_{B_{\bar{r}}(0)}\left|\left\langle\nabla u, V_{k+1}^{\mu}\right\rangle\right|^{p} d z \geq \bar{r}^{m-p} \eta .
\end{aligned}
$$

In particular, putting together Eqs. (4.27) and (4.28) we obtain:

$$
\lambda_{k+1} \leq \frac{(k+1) C_{6}(\sigma, m, p)}{\eta \bar{r}^{m-p}} \int_{B_{1}(0)}(\theta(y, \sigma)-\theta(y, 1)) d \mu(y),
$$

which is $(\mathrm{JNd})$.

\section{A Collection of Structural Lemmas}

This section is devoted to building a series of "quantitative" geometric results about $p$-minimizing mappings, describing the behavior of some special subsets of $\mathcal{S}(u)$. Analogous results for (approximate) 2-harmonic maps can be found in [14, Section 4], although stated with some differences.

In Lemmas 5.1 to 5.3, recall that $t_{a}, t_{b}$ are the structural constants introduced in Definition 2.8, describing some particular features of $\psi$. Moreover, in each of those results we implicitly assume that $u \in W^{1, p}(\Omega, \mathcal{N})$ is a $p$-energy minimizing map with energy bounded by $\Lambda$, and the constants we find are independent of $u$.

In the first lemma we convey this idea: consider the set of points in $B_{1}(0)$ at which $\theta[u]$ satisfies a suitable pinching condition; if it spans $\varrho$-effectively a $k$-dimensional plane $L$, then for some $\delta>0$ the stratum $\mathcal{S}_{\eta, \delta}^{k}(u)$ lies inside a fattening of $L$. See Definition 3.4 for the definition of a set effectively spanning a $k$-plane.

Lemma 5.1 Let $\varrho_{1}, \eta>0,0<\lambda_{1}<1$ and $0<\zeta<1$ be (small enough) constants. Define $c=c(\zeta) \doteq \frac{1}{2}(1-\zeta) t_{a}$, where $t_{a}$ is introduced in Definition 2.8. There exist constants $\delta_{0}, \varepsilon$ (depending on $m, p, \mathcal{N}, \Lambda$ and on the parameters just introduced) such that the following holds: if the set

$$
\mathcal{K} \doteq\left\{y \in B_{c r}(x) \mid \theta(y, r)-\theta\left(y, \lambda_{1} r\right)<\varepsilon\right\}
$$

spans $\varrho_{1} r$-effectively a k-plane $L$, then $\mathcal{S}_{\eta, \delta_{0} r}^{k}(u) \cap B_{c r}(x) \subset \mathcal{B}_{\varrho_{1} r}(L)$.

In the proof we drop the subscript 1 on $\varrho$ and $\lambda$; it was introduced so that lemma is easier to recall when we need it.

Proof Assume without loss of generality that $x=0, r=1$. We are thus assuming that 


$$
\mathcal{K}=\left\{y \in B_{c}(0) \mid \theta(y, 1)-\theta(y, \lambda)<\varepsilon\right\} \quad \text { spans } \varrho \text {-effectively } L \in \mathbf{H}^{k}\left(\mathbb{R}^{m}\right),
$$

with $\lambda$ and $\varrho$ fixed and $\varepsilon$ to be chosen. For a fixed point $w$ out of $\mathcal{B}_{\varrho}(L)$ we need to show that

$$
\tau^{p-m} \int_{B_{\tau}(w)}\left|\nabla_{V} u\right|^{p}<\eta
$$

for some $\tau \geq \delta_{0}$, with $\delta_{0}$ depending only on $m, p, \mathcal{N}, \Lambda, \eta, \varrho, \lambda, \zeta$, and for some $k+1$ dimensional plane $V$. Denote by $y_{0}, \ldots, y_{k}$ a set of points of $\mathcal{K}$ which $\varrho$-effectively span $L$.

Step 1 By Subtheorem 4.6.2, with $y \in \mathcal{K}, R_{1}=1, R_{2}=\lambda$ and $\max \{\lambda, 1-\zeta\} t_{a}<$ $R<t_{a}$, we have

$$
\int_{B_{R}(y)}|\nabla u(z)|^{p-2}|\langle\nabla u(z), y-z\rangle|^{2} d z \leq c_{1}(\theta(y, 1)-\theta(y, \lambda)) \leq c_{1} \varepsilon .
$$

Step 2 Notice that $B_{c}(0) \subset B_{R}(y)$ for any $y \in \mathcal{K}$ and $R$ as in the previous point; then for any such $y$ :

$$
\int_{B_{c}(0)}|\nabla u|^{p-2}|\langle\nabla u, y-z\rangle|^{2} d z \leq c_{1} \varepsilon .
$$

Define $\hat{L}$ to be the linear subspace associated to $L$. For any $v \in \hat{L}$ of norm 1, we have that

$$
v=\sum_{i=1}^{k} \alpha_{i}\left(y_{i}-y_{0}\right), \quad\left|\alpha_{i}\right| \leq c_{2}(m) .
$$

Hence, by a standard estimate,

$$
\begin{aligned}
\int_{B_{\tau}(w)}|\nabla u(z)|^{p-2}\left|\nabla_{v} u(z)\right|^{2} d z & \leq 2 \sum_{i} \alpha_{i}^{2} \int_{B_{c}(0)}|\nabla u|^{p-2}\left|\left\langle\nabla u, y_{i}-z\right\rangle\right|^{2} d z+ \\
& +2\left(\sum_{i} \alpha_{i}\right)^{2} \int_{B_{c}(0)}|\nabla u|^{p-2}\left|\left\langle\nabla u, z-y_{0}\right\rangle\right|^{2} d z \leq \\
& \leq c_{3}(m) \varepsilon .
\end{aligned}
$$

As a consequence, if $\left\{v_{1}, \ldots, v_{k}\right\}$ is an orthonormal basis of $\hat{L}$,

$$
\int_{B_{\tau}(w)}|\nabla u(z)|^{p-2} \sum_{i=1}^{k}\left|\nabla_{v_{i}} u(z)\right|^{2} d z \leq c_{4}(m) \varepsilon ;
$$


thus, along $k$ directions, we have some information that goes in the direction we need (recall that $\varepsilon$ is a constant we still have to choose).

Step 3 For points lying out of $\mathcal{B}_{\varrho}(L)$ we need to gain another direction of smallness, and we do it by considering the orthogonal direction to $L$ passing through $w$ : we can estimate the quantity

$$
\int_{B_{\tau}(w)}|\nabla u|^{p-2} \mid\left\langle\nabla u(z),\left.\frac{z-\pi_{L}(z)}{\left|z-\pi_{L}(z)\right|}\right|^{2} d z\right.
$$

using the same technique of the first point, and then exploit the triangle inequality to achieve an estimate on

$$
\int_{B_{\tau}(w)}|\nabla u|^{p-2}\left|\left\langle\nabla u(z), \frac{w-\pi_{L}(w)}{\left|w-\pi_{L}(w)\right|}\right\rangle\right|^{2} d z .
$$

More precisely: assume now that $\tau<\frac{\varrho}{2}$; define for the sake of simplicity $h(z) \doteq$ $\frac{z-\pi_{L}(z)}{\left|z-\pi_{L}(z)\right|}$, where $\pi_{L}$ is the orthogonal projection onto $L$. Similarly to what happened in the previous step, we have

$$
\begin{gathered}
\pi_{L}(z)=y_{0}+\sum_{i=1}^{k} \beta_{i}(z)\left(y_{i}-y_{0}\right), \quad\left|\beta_{i}(z)\right| \leq c_{5}(m) \\
\Longrightarrow \int_{B_{\tau}(w)}|\nabla u|^{p-2}\left|\left\langle\nabla u, z-\pi_{L}(z)\right\rangle\right|^{2} d z \leq c_{6}(m) \varepsilon
\end{gathered}
$$

so that

$$
\int_{B_{\tau}(w)}|\nabla u|^{p-2}\left|\left\langle\nabla u, \frac{z-\pi_{L}(z)}{\left|z-\pi_{L}(z)\right|}\right\rangle\right|^{2} d z \leq 4 c_{6}(m) \varepsilon \varrho^{2} .
$$

Moreover, for any $z \in B_{\tau}(w)$ we have that $z=w+\tau v^{\perp}+\tau v^{\top}$, with $v^{\top} \in \hat{L} \cap B_{1}(0)$, $v^{\perp} \in \hat{L}^{\perp} \cap B_{1}(0)$. In that case, the projection of $z$ onto $L$ can be written as $\pi_{L}(z)=$ $\pi_{L}(w)+\tau v^{\top}$. As a consequence, we can estimate

$$
\begin{aligned}
|h(z)-h(w)| & =\left|\frac{w-\pi_{L}(w)+\tau v^{\perp}}{\left|z-\pi_{L}(z)\right|}-\frac{w-\pi_{L}(w)}{\left|w-\pi_{L}(w)\right|}\right| \\
& =\left|\left(\frac{1}{\left|z-\pi_{L}(z)\right|}-\frac{1}{\left|w-\pi_{L}(w)\right|}\right)\left(w-\pi_{L}(w)\right)+\frac{\tau v^{\perp}}{\left|z-\pi_{L}(z)\right|}\right| \\
& \leq \frac{|| w-\pi_{L}(w)|-| z-\pi_{L}(z)||+\tau}{\left|z-\pi_{L}(z)\right|} \leq \frac{4 \tau}{\varrho} .
\end{aligned}
$$


To estimate the expression in (5.10), we exploit Eq. (5.13) and (5.14), the bound on $\theta(w, \tau)$ and the Cauchy-Schwarz inequality:

$$
\begin{aligned}
\int_{B_{\tau}(w)}|\nabla u|^{p-2}|\langle\nabla u(z), h(w)\rangle|^{2} d z \leq & 2 \int_{B_{\tau}(w)}|\nabla u|^{p-2}|\langle\nabla u, h(z)\rangle|^{2} d z+ \\
& +2 \int_{B_{\tau}(w)}|\nabla u|^{p-2}|\langle\nabla u, h(w)-h(z)\rangle|^{2} d z \leq \\
\leq & 4 c_{6}(m) \varepsilon \varrho^{2}+\frac{32 \Lambda}{\varrho^{2}} \tau^{2-(p-m)} .
\end{aligned}
$$

Step 4 Putting together the previous steps, we consider $V=\hat{L} \oplus h(w)$ : a simple computation gives:

$$
\begin{aligned}
\tau^{p-m} \int_{B_{\tau}(w)}\left|\nabla_{V} u\right|^{p} d z \leq & \tau^{p-m} \int_{B_{\tau}(w)}|\nabla u(z)|^{p-2} \sum_{i=1}^{k}\left|\nabla_{v_{i}} u(z)\right|^{2} d z \\
& +\tau^{p-m} \int_{B_{\tau}(w)}|\nabla u|^{p-2}|\langle\nabla u(z), h(w)\rangle|^{2} d z \\
\leq & c_{4}(m) \varepsilon \tau^{p-m}+4 c_{6}(m) \varrho^{2} \varepsilon \tau^{p-m}+\frac{32 \Lambda}{\varrho^{2}} \tau^{2}
\end{aligned}
$$

Thus, in order to conclude, we only need to choose $\tau$ (and thus $\delta_{0}$ ) so that the last term is smaller than $\frac{\eta}{2}$, and then choose $\varepsilon$ such that also the sum of the first two pieces is smaller than $\frac{\eta}{2}$.

The upcoming lemma says the following: if we have a set of points that satisfy a suitable pinching condition on $\theta$, and they effectively span a $k$-subspace $L$, then all the points of $L$ inherit a (possibly weaker) pinching condition. This can be seen as a further quantitative version of Corollary 2.10 (it is indeed applied to the limit of a contradicting sequence).

Lemma 5.2 Let $\varrho_{2}, \lambda_{2}, \Lambda, \zeta>0$. Let $0<c<\frac{1}{2} t_{b}$. There exists a constant $\delta_{1}\left(\varrho_{2}, \lambda_{2}, \Lambda, \zeta, c\right)$ such that the following holds: if $\theta(y, r) \leq E$ for all $y \in B_{c r}(x) \cap \mathcal{S}$ (with $\mathcal{S} \subset \Omega$ and $E \leq \Lambda$ ), and the set

$$
\mathcal{H} \doteq\left\{y \in B_{c r}(x) \cap \mathcal{S} \mid \theta\left(x, \lambda_{2} r\right)>E-\delta_{1}\right\}
$$

spans $\varrho_{2} r$-effectively a $k$-space $L$, then we have

$$
\theta\left(z, \lambda_{2} r\right)>E-\zeta
$$

for all $z \in B_{c r}(x) \cap L$.

In the proof we drop the subscript 2 on $\varrho$ and $\lambda$; it was introduced so that lemma is easier to recall when we need it. 
Proof Assume $x=0, r=1$. If the statement is false, one can find a sequence of $p$-minimizing maps $\left\{u_{i}\right\}_{i}, k+1$ sequences of points $\left\{y_{i j}\right\}_{i \in \mathbb{N}}$ in $B_{c}(0) \cap \mathcal{S}$ (with $j=1, \ldots, k+1)$, and a further sequence $\left\{z_{i}\right\}_{i}$ in $B_{c}(0)$ such that:

- $\left\{y_{i j}\right\}_{j=1}^{k+1}$ spans $\varrho$-effectively a $k$-space $L$ (which can be assumed to be the same for all $i$ ).

- $\theta\left[u_{i}\right]\left(y_{i j}, \lambda\right)>E-\frac{1}{i}$, and $\theta\left[u_{i}\right]\left(y_{i j}, 1\right) \leq E$.

- $\theta\left[u_{i}\right]\left(z_{i}, \lambda\right)<E-\zeta$, and $z_{i} \in L$.

Up to subsequences, $\left\{u_{i}\right\}$ converges in $W^{1, p}$ to a $p$-minimizing map $\bar{u}, y_{i j} \rightarrow \bar{y}_{j} \in$ $\bar{B}_{c}(0)$, and $z_{i} \rightarrow \bar{z} \in \bar{B}_{c}(0) \cap L$. Moreover, the set of points $\left\{\bar{y}_{j}\right\}_{j=1}^{k+1}$ still spans $L$, and we have $\theta[\bar{u}]\left(\bar{y}_{j}, \lambda\right)=E$, which implies

$$
\theta[\bar{u}]\left(\bar{y}_{j}, 1\right)-\theta[\bar{u}]\left(\bar{y}_{j}, \lambda\right)=0 .
$$

Thus $\bar{u}$ is $L$-invariant in $\bar{B}_{c}(0)$ by Corollary 2.10; so in particular both $\theta[\bar{u}](\bar{z}, \lambda)=E$ and $\theta[\bar{u}](\bar{z}, \lambda) \leq E-\zeta$ should hold.

Finally, a result which states that the lack of almost invariance spreads uniformly along pinched points. This is yet another quantitative rephrasing of the fact that if $\theta(\cdot, r)-\theta(\cdot, \lambda r)=0$ at two different (close) points, then $u$ is invariant along the direction connecting them.

Lemma 5.3 Let $\lambda>0, \sigma_{0} \in\left(0, \frac{1}{2} t_{b}\right), \kappa_{0} \in(0,1)$. There exists a constant $\varepsilon$ such that the following holds. If the following conditions are satisfied by a pair of points $x, y$ :

1. $|x-y|<\frac{1}{2} t_{b} r$

2. $\theta(x, r)-\theta(x, \lambda r)<\varepsilon$;

3. $\theta(y, r)-\theta(y, \lambda r)<\varepsilon$;

and $u$ is not $(\eta, k)$-invariant in $B_{\sigma r}(x)$ for some $\sigma_{0} \leq \sigma \leq \frac{1}{2} t_{b}$, then $u$ is not $\left(\kappa_{0} \eta, k\right)$ invariant in $B_{\sigma r}(y)$.

Proof Assume $x=0, r=1$. By contradiction, there exist: a sequence $\left\{u_{i}\right\}_{i}$ of $p$-minimizing maps, a sequence $\left\{y_{i}\right\}_{i}$ of points in $B_{\frac{1}{2} t_{b}}(0)$ and a sequence $\left\{\sigma_{i}\right\}_{i}$ in $\left[\sigma_{0}, \frac{1}{2} t_{b}\right]$ such that:

$$
\begin{aligned}
\theta\left[u_{i}\right](0,1)-\theta\left[u_{i}\right](0, \lambda) & <\frac{1}{i}, \\
\theta\left[u_{i}\right]\left(y_{i}, 1\right)-\theta\left[u_{i}\right]\left(y_{i}, \lambda\right) & <\frac{1}{i}, \\
\sigma_{i}^{p-m} \int_{B_{\sigma_{i}}(0)}\left|\nabla_{L} u_{i}\right|^{p} & >\eta \quad \forall L \in \mathbf{G}^{k}\left(\mathbb{R}^{m}\right), \\
\sigma_{i}^{p-m} \int_{B_{\sigma_{i}}\left(y_{i}\right)}\left|\nabla_{\tilde{L}} u_{i}\right|^{p} & <\kappa_{0} \eta \quad \exists \tilde{L} \in \mathbf{G}^{k}\left(\mathbb{R}^{m}\right) .
\end{aligned}
$$

Up to subsequences, they converge, respectively, to a $p$-minimizing map $\bar{u}$ (in $W^{1, p}$ ), to a point $\bar{y} \in \bar{B}_{\frac{1}{2} t_{b}}(0)$ and to a number $\bar{\sigma} \in\left[\sigma_{0}, \frac{1}{2} t_{b}\right]$. Moreover, due to Eqs. (5.20) 
and (5.21) and by the $L^{p}$-convergence of gradients, $\bar{u}$ is 0 -homogeneous with respect to both $x=0$ and $\bar{y}$, thus it is invariant along the direction $\frac{\bar{y}}{|\bar{y}|}$ in $B_{\frac{1}{2} t_{b}}(0)$. Again by the fact that $u_{i} \rightarrow \bar{u}$ in $W^{1, p}(\Omega, \mathcal{N})$, however, we also have that

$$
\begin{gathered}
\bar{\sigma}^{p-m} \int_{B_{\sigma}(0)}\left|\nabla_{\tilde{L}} \bar{u}\right|^{p} \geq \eta \\
\bar{\sigma}^{p-m} \int_{B_{\sigma}(\bar{y})}\left|\nabla_{\tilde{L}} \bar{u}\right|^{p} \leq \kappa_{0} \eta ;
\end{gathered}
$$

this contradicts the fact that the two left hand sides should be equal (by translation invariance of $\bar{u}$ ).

Remark (Assumptions on $\psi$, again) The structural constants $t_{a}$ and $t_{b}$ we introduced in Definition 2.8 were broadly used in these last few lemmas. In practice, the key feature (of $\psi$ ) we need for our purposes is the possibility to work handily in the ball $B_{1}(0)$ (so for example we require that 1 is an admissible value for $c(\zeta)$ in Lemma 5.1). In the end, with the choice $t_{a}>2$ done in Definition 2.8, we can apply Lemmas 5.1 and 5.2 with $c=1$ and Lemma 5.3 with $|x-y|<r$.

\section{Covering Arguments}

In the sequel, if $B=B_{r}(x)$ and $k$ is a positive number, we denote by $k B$ the ball $B_{k r}(x)$. We first give two useful definitions of "sets of points satisfying a pinched condition"; we have (more or less) already used both of them in Sect. 5.

Definition 6.1 Let $u$ be a $p$-minimizing map, $x \in B_{1}(0), r>0$. Assume $E, \lambda, \delta>0$ are fixed, and $\mathcal{S} \subset B_{1}(0)$. We define

$$
\begin{aligned}
& \mathcal{H}(x, r)=\mathcal{H}_{E, \delta, \lambda}^{\mathcal{S}}(x, r) \doteq\left\{y \in B_{r}(x) \cap \mathcal{S} \mid \theta(y, \lambda r)>E-\delta\right\} \\
& \mathcal{K}(x, r)=\mathcal{K}_{\delta, \lambda}^{\mathcal{S}}(x, r) \doteq\left\{y \in B_{r}(x) \cap \mathcal{S} \mid \theta(y, r)-\theta(y, \lambda r)<\delta\right\} .
\end{aligned}
$$

If $B=B_{r}(x)$, we also denote by $\mathcal{H}_{B}, \mathcal{K}_{B}$ the sets $\mathcal{H}(x, r), \mathcal{K}(x, r)$ respectively.

It is clear that, if all the parameters appearing are fixed, and $u$ is such that $\theta(y, r) \leq$ $E$ for all $y \in B_{r}(x)$, then $\mathcal{H}(x, r) \subset \mathcal{K}(x, r)$. As a consequence, whenever $\mathcal{H} \varrho$ effectively spans a $k$-subspace, also $\mathcal{K}$ trivially does. Heuristically $\mathcal{H}$ should be thought as a set of pinched points at which $\theta(y, \lambda r)$ has a value which is close to the maximum possible.

Notations and map of the constants. This will be the context for the whole section:

$-m, \mathcal{N}, p, \Lambda$ are fixed as in the previous sections (respectively: dimension of the domain, target manifold, exponent for the energy, upper bound on the $p$-energy).

$-u \in W^{1, p}(\Omega, \mathcal{N})$ is a $p$-minimizing harmonic map with $p$-energy bounded by $\Lambda$.

- We let $\varrho \in(0,1)$ be a fixed constant, and $r=\varrho^{\hat{\jmath}}$ for some $\hat{\jmath} \in \mathbb{N}_{\geq 1}$. The radius $r$ will be the scale parameter for the singular stratification, up to a constant. The 
constant $\varrho$ will be arbitrary in the first covering, and will be then suitably selected in the construction of the second covering.

$-\eta>0$ is the (fixed) closeness parameter for the stratification.

$-k \in\{1, \ldots, m\}$ is the dimension parameter for the stratification.

$-\zeta>0$ is a constant used for the pinching condition on $\theta$. It will be arbitrary in the construction of the first covering, then selected in Proposition 6.4.

$-\delta_{0}$ and $\varepsilon$ are the constants produced by Lemma 5.1 when $\varrho_{1}=\lambda_{1}=\frac{1}{5} \varrho, \eta$ is the already chosen closeness parameter and $\zeta$ is chosen such that $c(\zeta)=1 ; \delta_{1}$ is the constant produced by Lemma 5.2 when $\varrho_{2}=\lambda_{2}=\frac{1}{5} \varrho, \zeta$ is the arbitrary parameter introduced above and $c=1$. We fix $\delta=\delta(\varrho, \eta, \zeta) \doteq \min \left\{\varepsilon, \delta_{1}\right\}$.

$-\mathcal{S}$ is a subset of the stratum $\mathcal{S}_{\eta, \delta_{0} r}^{k}(u) \cap B_{1}(0)$.

$-0<E \leq \Lambda$ is such that $\theta(x, 1) \leq E$ for all $x \in B_{1}(0) \cap \mathcal{S}$.

The goal of the upcoming constructions will be to build a "controlled" covering of $\mathcal{S}$. In words, the ultimate goal will be to cover $\mathcal{S}$ with balls $B$ satisfying the following:

1. The sum of the $k^{\text {th }}$ powers of the radii is bounded by a universal constant.

2. Up to rescaling by a fixed constant, the balls are pairwise disjoint.

3. Either the radius of $B$ is less than or equal to the fixed radius $r$; or the (normalized) $p$-energy in $B$ is lower than the "maximal initial $p$-energy" $E$ by a fixed amount $\delta$ ( $E$ and $\delta$ were introduced in the previous list of constants). In the latter case, we say that $B$ satisfies a uniform energy drop condition (see the below Definition 6.2).

This will be achieved in Proposition 6.5. Once we have this, we can then apply the same reasoning to each of the balls where the $p$-energy drops uniformly (while keeping the other balls as they are). At each step other balls of radius $\leq r$ are produced, while the $p$-energy continues to drop uniformly in all the other balls. The procedure lasts a finite number of steps, until there's no energy left: indeed the total initial $p$-energy was bounded by a fixed constant $\Lambda$. This is the content of Sect. 7.1. Let's give a precise definition of energy drop:

Definition 6.2 We say that a ball $B$ with radius $r_{B}$ satisfies the uniform $(\lambda, \delta)$-energy drop condition if

$$
\theta\left(y, \lambda r_{B}\right) \leq E-\delta \quad \text { for all } y \in \mathcal{S} \cap B
$$

From now on $\lambda=\frac{1}{5}$ and $\delta$ will be fixed ( $\delta$ as in the Map of the Constants above), so we omit them and simply say "uniform energy drop".

At first, we are only able to reach a partial result: we don't manage to fully get a uniform energy drop condition on the balls of the covering; but we can show that, in each ball, the points for which the $p$-energy does not drop uniformly lie close to a $(k-1)$-plane (so in the end they can be controlled very efficiently). This is the content of the next subsection. 


\subsection{First Covering}

Recall that $r=\varrho^{\hat{\jmath}}$ is the scale parameter of the singular stratum we are considering. Here $\varrho>0$ is a fixed parameter and $\hat{\jmath} \in \mathbb{N}$ : we are allowed to work with constants which depend on $\varrho$, but not on $\hat{\jmath}$.

Construction of the first covering We construct a covering $\mathcal{F}$ of $\mathcal{S}$ with the following properties:

1. $\mathcal{F}=\mathcal{G}_{0} \cup \cdots \cup \mathcal{G}_{\hat{\jmath}-1} \cup \mathcal{E}_{\hat{\jmath}}$. If $B \in \mathcal{G}_{h}$, then $B=B_{\varrho^{h}}(x)$ for some $x$. If $B \in \mathcal{E}_{\hat{\jmath}}$, then $B=B_{\varrho^{\hat{\jmath}}}(x)$ for some $x$. Mnemonic rule: when the construction of $\mathcal{F}$ is complete, the subcovering $\mathcal{E}_{\hat{\jmath}}$ is made of balls with radius equal to $r$, while the subcoverings labeled with $\mathcal{G}$ are made of balls with radius greater than $r$.

2. If $B_{\varrho^{h}}(x) \in \mathcal{G}_{h}$ with $0 \leq h \leq \hat{\jmath}-1$, then $\mathcal{H}\left(x, \varrho^{h}\right) \subset \mathcal{B}_{\frac{1}{5} \varrho^{h+1}}(V)$ for some $(k-1)$-affine subspace $V \in \mathbf{H}^{k-1}\left(\mathbb{R}^{m}\right)$; here $\mathcal{H}=\mathcal{H}_{E, \delta, \frac{1}{5}}^{\mathcal{S}}$.

3. If $B, B^{\prime} \in \mathcal{F}$ and $B \neq B^{\prime}$, then $\frac{1}{5} B \cap \frac{1}{5} B^{\prime}=\varnothing$.

4. If $B_{r_{x}}(x) \in \mathcal{G}_{1} \cup \cdots \cup \mathcal{G}_{\hat{\jmath}-1} \cup \mathcal{E}_{\hat{\jmath}}$, then

$$
\begin{aligned}
& \theta\left(x, \frac{1}{5} r_{x}\right)>E-\zeta, \\
& x \in \mathcal{S}_{\frac{1}{2} \eta, \delta_{0} r_{x}}^{k}(u) .
\end{aligned}
$$

The strategy will be to apply inductively the lemmas from Sect. 5 at different scales. We thus proceed inductively on $j \in\{0, \ldots, \hat{\jmath}\}$.

Step 1, case A If $\mathcal{H}(0,1)$ is contained in $\mathcal{B}_{\frac{1}{5} \varrho}(V)$ with $V \in \mathbf{H}^{k-1}\left(\mathbb{R}^{m}\right)$, then we define $\mathcal{G}_{0} \doteq\left\{B_{1}(0)\right\}$. The other subcoverings are left empty, and the process stops here.

Step 1, case B Otherwise, $\mathcal{H}(0,1)$ spans $\frac{1}{5} \varrho$-effectively a $k$-space $L(0,1) \in \mathbf{H}^{k}\left(\mathbb{R}^{m}\right)$. Thus, by Lemma 5.1 with $\lambda_{1}=\varrho_{1}=\frac{1}{5} \varrho, \mathcal{S} \cap B_{1}(0)$ is contained in $\mathcal{B}_{\frac{1}{5} \varrho}(L(0,1))$. By Lemma 5.2, with $\lambda_{2}=\varrho_{2}=\frac{1}{5} \varrho$, for any $z \in L(0,1) \cap B_{1}(0)$ we have

$$
\theta\left(z, \frac{1}{5} \varrho\right)>E-\zeta .
$$

If $\zeta$ is small enough (smaller than a constant depending on $m, p, \eta, \varrho$ ), by Lemma 5.3 with $\kappa_{0}=\frac{1}{2}, \lambda=\frac{1}{5} \varrho$ and $\sigma=\delta_{0} \varrho$, for any $z \in L(0,1) \cap B_{1}(0)$ we have $z \in \mathcal{S}_{\frac{1}{2} \eta, \delta_{0} \varrho}^{k}(u)$ (because $\mathcal{S}_{\eta, \delta_{0} \varrho}^{k}(u) \supset \mathcal{S}_{\eta, \delta_{0} r}^{k}(u)$ ). Cover $\mathcal{S} \cap B_{1}(0)$ with balls of radius $\varrho$ with centers in $L(0,1)$ and such that $\frac{1}{5} B \cap \frac{1}{5} B^{\prime}=\varnothing$ if $B \neq B^{\prime}$. Call $\mathcal{E}_{1}$ this covering.

If $\hat{\jmath}=1$, i.e. the final radius $r$ we want to reach is $\varrho^{1}$, then we can stop here the procedure. Otherwise, assume that $B \doteq B_{\varrho}(x) \in \mathcal{E}_{1}$ is a ball produced by Step 1, case B.

Step 2, case A If $\mathcal{H}(x, \varrho) \subset \mathcal{B}_{\frac{1}{5} \varrho^{2}}(V)$ for some $V \in \mathbf{H}^{k-1}\left(\mathbb{R}^{m}\right)$, then $B$ is one of the balls that we want to keep in our final covering $\mathcal{F}$; we define

$$
\mathcal{G}_{1} \doteq\left\{B_{\varrho}(x) \in \mathcal{E}_{1} \mid \mathcal{H}(x, \varrho) \subset \mathcal{B}_{\frac{1}{5} \varrho^{2}}(V) \text { for some } V \in \mathbf{H}^{k-1}\left(\mathbb{R}^{m}\right)\right\}
$$


Step 2, case B If instead $B \notin \mathcal{G}_{1}$, this means that $\mathcal{H}(x, \varrho)$ spans $\frac{1}{5} \varrho^{2}$-effectively a $k$-space $L(x, \varrho)$. Thus, applying Lemmas 5.1 to 5.3 with the same constants as in Step 1, Case B, we get:

1. $\mathcal{S} \cap B_{\varrho}(x) \subset \mathcal{B}_{\frac{1}{5} \varrho^{2}}(L(x, \varrho))$ for some $L(x, \varrho) \in \mathbf{H}^{k}\left(\mathbb{R}^{m}\right)$;

2. $\theta\left(z, \frac{1}{5} \varrho^{2}\right)>E-\zeta$ for all $z \in L(x, \varrho) \cap B_{\varrho}(x)$;

3. $z \in \mathcal{S}_{\frac{1}{2} \eta, \delta_{0} \varrho^{2}}^{k}(u)$ for all $z \in L(x, \varrho) \cap B_{\varrho}(x)$.

Now we cover $\mathcal{S} \cap B_{\varrho}(x) \backslash \bigcup \mathcal{G}_{1}$ with balls of radius $\varrho^{2}$ such that for any pair $B \neq B^{\prime}$ of such balls we have $\frac{1}{5} B \cap \frac{1}{5} B^{\prime}=\varnothing$ and $\frac{1}{5} B \subset B_{\varrho}(x) \backslash \bigcup \mathcal{G}_{1}$; define $\mathcal{E}_{2, x}$ such a covering. Define

$$
\mathcal{E}_{2} \doteq \bigcup\left\{\mathcal{E}_{2, x} \mid B(x, \varrho) \in \mathcal{E}_{1} \backslash \mathcal{G}_{1}\right\}
$$

This concludes Step 2.

After the $j^{\text {th }}$ step, we have:

- $j$ families of balls $\mathcal{G}_{0}, \ldots, \mathcal{G}_{j-1}$, with the following properties: if $B \in \mathcal{G}_{h}$ then $B=B_{\varrho^{h}}(x)$ for some $x$, and $\mathcal{H}\left(x, \varrho^{h}\right)$ is contained in $\mathcal{B}_{\frac{1}{5} \varrho^{h+1}}(V)$ for some $V \in$ $\mathbf{H}^{k-1}\left(\mathbb{R}^{m}\right)$;

- A family $\mathcal{E}_{j}$ of balls of radius $\varrho^{j}$.

If $j=\hat{j}$, then we are done. Otherwise, we proceed in the same fashion. Let $B=$ $B_{\varrho^{j}}(x) \in \mathcal{E}_{j}$.

Step $j+1$, case A. If $\mathcal{H}\left(x, \varrho^{j}\right) \subset \mathcal{B}_{\frac{1}{5} \varrho^{j+1}}(V)$ for some $V \in \mathbf{H}^{k-1}\left(\mathbb{R}^{m}\right)$, then $B$ is one of the balls that we want to keep in our final covering $\mathcal{F}$; we define

$$
\mathcal{G}_{j} \doteq\left\{B_{\varrho^{j}}(x) \in \mathcal{E}_{j} \mid \mathcal{H}\left(x, \varrho^{j}\right) \subset \mathcal{B}_{\frac{1}{5} \varrho^{j+1}}(V) \text { for some } V \in \mathbf{H}^{k-1}\left(\mathbb{R}^{m}\right)\right\} \text {. }
$$

Step $j+1$, case B. If instead $B \notin \mathcal{G}_{j}$, this means that $\mathcal{H}\left(x, \varrho^{j}\right)$ spans $\frac{1}{5} \varrho^{j+1}$-effectively a $k$-space $L\left(x, \varrho^{j}\right)$. Thus, applying Lemmas 5.1 to 5.3 with the same constants as in Case B of the previous steps, we get:

1. $\mathcal{S} \cap B \subset \mathcal{B}_{\frac{1}{5} \varrho^{j+1}}\left(L\left(x, \varrho^{j}\right)\right)$ for some $L\left(x, \varrho^{j}\right) \in \mathbf{H}^{k}\left(\mathbb{R}^{m}\right)$;

2. $\theta\left(z, \frac{1}{5} \varrho^{j+1}\right)>E-\zeta$ for all $z \in L\left(x, \varrho^{j}\right) \cap B_{\varrho^{j}}(x)$;

3. $z \in \mathcal{S}_{\frac{1}{2} \eta, \delta_{0} \varrho^{j+1}}^{k}(u)$ for all $z \in L\left(x, \varrho^{j}\right) \cap B_{\varrho^{j}}(x)$.

Now we cover $\mathcal{S} \cap B_{\varrho^{j}}(x) \backslash \bigcup_{h \leq j} \bigcup \mathcal{G}_{h}$ with balls of radius $\varrho^{j+1}$ such that for any pair $B \neq B^{\prime}$ of such balls we have $\frac{1}{5} B \cap \frac{1}{5} B^{\prime}=\varnothing$ and $\frac{1}{5} B \subset B_{\varrho}(x) \backslash \bigcup_{h \leq j} \bigcup \mathcal{G}_{h}$; define $\mathcal{E}_{j+1, x}$ such a covering. Define

$$
\mathcal{E}_{j+1} \doteq \bigcup\left\{\mathcal{E}_{j+1, x} \mid B(x, \varrho) \in \mathcal{E}_{j} \backslash \mathcal{G}_{j}\right\}
$$

Iterating the procedure until $\hat{\jmath}$, we obtain the desired construction. 
Definition 6.3 If $\mathcal{F}=\mathcal{G}_{0} \cup \cdots \cup \mathcal{G}_{\hat{\jmath}-1} \cup \mathcal{E}_{\hat{\jmath}}$ is the covering just constructed, define the following sets of centers:

$$
\begin{aligned}
\mathcal{D}_{h} & \doteq\left\{x \in B_{1}(0) \mid B_{\varrho^{h}}(x) \in \mathcal{G}_{h}\right\}, \quad 0 \leq h \leq \hat{\jmath}-1 \\
\mathcal{D}_{\hat{\jmath}} & \doteq\left\{x \in B_{1}(0) \mid B_{\varrho^{\hat{\jmath}}}(x) \in \mathcal{E}_{\hat{\jmath}}\right\} \\
\mathcal{C} & \doteq \mathcal{D}_{0} \cup \cdots \cup \mathcal{D}_{\hat{\jmath}-1} \cup \mathcal{D}_{\hat{\jmath}} \\
\mathcal{C}_{\ell} & \doteq \mathcal{D}_{\hat{\jmath}-\ell} \cup \cdots \cup \mathcal{D}_{\hat{\jmath}}, \quad 0 \leq \ell \leq \hat{\jmath} .
\end{aligned}
$$

Moreover, if $x \in \mathcal{C}$, we will also denote by $r_{x}$ the radius of the ball centered at $x$ which is contained in $\mathcal{F}$. Notice that

$$
\mathcal{C}_{\ell}=\left\{x \in \mathcal{C} \mid r_{x} \leq \varrho^{\hat{j}-\ell}\right\}
$$

and $\mathcal{C}=\mathcal{C}_{\hat{\jmath}} \subset \mathcal{C}_{\hat{\jmath}-1} \subset \cdots \subset \mathcal{C}_{1} \subset \mathcal{C}_{0}=\mathcal{D}_{\hat{\jmath}}$

The next step is probably the most important of the whole construction: indeed, we show that we have a control on the $k^{\text {th }}$ powers of the radii of the balls in $\mathcal{F}$. Here is where the refined techniques of Sect. 4 become involved: we use Reifenberg Theorem 4.4 to achieve the final estimate, and Theorem 4.6 to check Reifenberg's hypothesis. Unfortunately, the proof is a bit intricate: we split it in several subtheorems.

Remark From now on, we will assume without loss of generality that $\varrho$ is of the form $5^{-\kappa}$ for some $\kappa \in \mathbb{N}$. This does not affect in any way the general procedure (at some point we will choose $\varrho$ as an arbitrary number smaller than a certain constant) and simplifies a bit some computations.

Proposition 6.4 (Volume estimates) Let $\mathcal{F}=\left\{B_{r_{x}}(x)\right\}_{x \in \mathcal{C}}$ be the covering constructed in the previous paragraph. Recall that $\varrho, \eta, \zeta, E>0$ are fixed constants. If $\zeta>0$ and $\varrho>0$ are chosen small enough, there exists a constant $C_{I}=C_{I}(m, \varrho)$ such that

$$
\sum_{x \in \mathcal{C}} r_{x}^{k} \leq C_{I}
$$

By Reifenberg Theorem 4.4, the estimate (6.14) is achieved if the condition

$$
\int_{B_{\tau}(w)} \int_{0}^{\tau} \beta_{\mu}^{k}(y, s)^{2} \frac{d s}{s} d \mu(y)<\delta_{R} \tau^{k}
$$

holds for any ball $B_{\tau}(w)$ with $w \in B_{1}(0)$ and $0<\tau<1$ (or $0<\tau<\tau_{\max }$ for some $\tau_{\max }$, at the only price of worsening the constants involved). Here $\mu \doteq \sum_{x \in \mathcal{C}} r_{x}^{k} \delta_{x}$. For any $0 \leq h \leq \hat{\jmath}$, we now consider the measure $\mu_{h}$ associated to the set of centers $\mathcal{C}_{h}$ defined in Definition 6.3: $\mu_{h} \doteq \sum_{x \in \mathcal{C}_{h}} r_{x}^{k} \delta_{x}$. Clearly $\mu=\mu_{\hat{\jmath}}$; first of all, we state a very elementary "induction property" of the measures $\mu_{h}$. 
Subtheorem 6.4.1 Let $h \in\{0, \ldots, \hat{\jmath}-1\}$. Let $C_{\text {in }}>0$ be a constant. Assume without loss of generality $\varrho<\frac{1}{10}$. Assume that, for all $x \in B_{1}(0)$ and all $s \in\left[\frac{1}{5} \varrho^{\hat{\jmath}}, \varrho^{\hat{\jmath}-h}\right]$, it holds

$$
\mu_{h}\left(B_{S}(x)\right) \leq C_{i n} s^{k}
$$

Then there exists a constant $C_{f}$ depending only on $C_{i n}, \varrho$ and $m$ such that

$$
\mu_{h+1}\left(B_{s}(x)\right) \leq C_{f} s^{k}
$$

whenever one of the following holds:

(i) $B_{s}(x) \cap\left(\mathcal{C}_{h+1} \backslash \mathcal{C}_{h}\right)=\varnothing$ and $s \in\left[\frac{1}{5} \varrho^{\hat{\jmath}}, 2 \varrho^{\hat{\jmath}-(h+1)}\right]$.

(ii) $B_{s}(x)$ contains points of $\mathcal{C}_{h+1} \backslash \mathcal{C}_{h}$ and $s \in\left[2 \varrho^{\hat{\jmath}-h}, 2 \varrho^{\hat{\jmath}-(h+1)}\right]$.

Remark We could obviously state the same property with more general constants in front of the radii involved. This is however the form we will need: notice that the "upper bound" for the radius gains a factor 2 .

Proof Fix $x \in B_{1}(0)$ and $s \in\left[\frac{1}{5} \varrho^{\hat{\jmath}}, 2 \varrho^{\hat{\jmath}-(h+1)}\right]$. We can split $\mu_{h+1}\left(B_{S}(x)\right)$ as

$$
\begin{aligned}
\mu_{h+1}\left(B_{S}(x)\right) & =\mu_{h}\left(B_{S}(x)\right)+\sum_{\substack{z \in \mathcal{C}_{h+1} \backslash \mathcal{C}_{h} \\
z \in B_{s}(x)}} \varrho^{k(\hat{\jmath}-h-1)} \\
& =\mu_{h}\left(B_{S}(x)\right)+\varrho^{k(\hat{\jmath}-h-1)} \operatorname{card}\left(B_{S}(x) \cap \mathcal{C}_{h+1} \backslash \mathcal{C}_{h}\right) .
\end{aligned}
$$

Now:

- If $B_{S}(x) \cap\left(\mathcal{C}_{h+1} \backslash \mathcal{C}_{h}\right)=\varnothing$ and $s \in\left[\frac{1}{5} \varrho^{\hat{\jmath}}, \varrho^{\hat{\jmath}-h}\right]$, then the first term is smaller than or equal to $C_{i n} s^{k}$ by assumption; the second term is trivially zero.

- If $s \in\left[\varrho^{\hat{\jmath}-h}, 2 \varrho^{\hat{\jmath}-h-1}\right]$, then: we can cover $B_{S}(x) \cap \operatorname{supp}\left(\mu_{h}\right)$ with a controlled number $c_{1}(\varrho, m)$ of balls centered in $\mathcal{C}_{h}$ with radius $\varrho^{\hat{\jmath}-h}$, so that we obtain:

$$
\mu_{h}\left(B_{S}(x)\right) \leq c_{1} C_{i n} \varrho^{k(\hat{\jmath}-h)} \leq c_{1} C_{i n} s^{k}
$$

moreover, the number $\operatorname{card}\left(B_{S}(x) \cap\left(\mathcal{C}_{h+1} \backslash \mathcal{C}_{h}\right)\right)$ is also bounded by a constant $c_{2}(\varrho, m)$, because balls centered in $\left(\mathcal{C}_{h+1} \backslash \mathcal{C}_{h}\right)$ with radius $\frac{1}{5} \varrho^{\hat{\jmath}-h-1}$ do not contain points of $\mathcal{C}$ other then their center. Thus

$$
\varrho^{k(\hat{\jmath}-h-1)} \operatorname{card}\left(B_{S}(x) \cap \mathcal{C}_{h+1} \backslash \mathcal{C}_{h}\right) \leq \frac{c_{2}}{\varrho^{k}} s^{k} .
$$

By choosing $C_{f} \doteq \max \left\{C_{i n}, c_{1} C_{i n}+\frac{c_{2}}{\varrho^{k}}\right\}$ we get the result.

The next step is to prove that the estimate Eq. (6.16) actually holds when $h=0$. 
Subtheorem 6.4.2 There exists a constant $C_{0}(\varrho, m)$ such that: for any $x \in B_{1}(0)$ and $s \in\left[\frac{1}{5} \varrho^{\hat{\jmath}}, \varrho^{\hat{\jmath}}\right]$,

$$
\mu_{0}\left(B_{s}(x)\right) \leq C_{0} s^{k}
$$

Proof An argument already used in Subtheorem 6.4.1: if $x \neq y \in \mathcal{C}_{0}$, then $B_{\frac{1}{5} \varrho^{\hat{\jmath}}}(x)$ and $B_{\frac{1}{5} \varrho^{\hat{j}}}(y)$ are disjoint, thus the number of such centers contained in $B_{S}(x)$ is bounded by a constant (the same $c_{2}(\varrho, m)$ as in the previous proof). Thus

$$
\mu_{0}\left(B_{s}(x)\right) \leq c_{2}(\varrho, m) \varrho^{\hat{\jmath} k} \leq 5^{k} c_{2} s^{k}
$$

which is what we needed.

It may seem that, having an inductive step and a base step, we could already get the volume estimate we need. The problem is that we are applying Subtheorem 6.4.1 with an initial constant $C_{i n}$ that keeps getting bigger at any step; instead, we would need in the end a universal constant that only depends on $\varrho$ and $m$, since the number of steps is not fixed a priori, and we don't want our constants to depend on it. Here is where Reifenberg Theorem comes into play. The trick will be to prove that the estimate

$$
\int_{B_{\tau}(w)} \int_{0}^{\tau} \beta_{\mu_{h}}^{k}(y, s)^{2} \frac{d s}{s} d \mu_{h}(y)<\delta_{R} \tau^{k}
$$

holds for any $\mu_{h}$.

Subtheorem 6.4.3 Let $\varrho>0$ (small enough) and $C_{f}>0$ be fixed constants; $\eta, E, \mathcal{S}$ as before. There exists a constant $\zeta=\zeta\left(\varrho, C_{f}, m, p, \eta\right)$ such that the following holds. Assume that $\mathcal{F}$ is the covering of $\mathcal{S}$ associated to the constant $\zeta$, and that $\mu_{h}$ verifies the conclusion of Subtheorem 6.4.1, i.e.: the estimate

$$
\mu_{h}\left(B_{s}(x)\right) \leq C_{f} s^{k}
$$

holds whenever one of the following holds:

(i) $B_{s}(x) \cap\left(\mathcal{C}_{h} \backslash \mathcal{C}_{h-1}\right)=\varnothing$ and all $s \in\left[\frac{1}{5} \varrho^{\hat{\jmath}}, 2 \varrho^{\hat{\jmath}-h}\right]$.

(ii) $B_{s}(x)$ contains a point of $\mathcal{C}_{h} \backslash \mathcal{C}_{h-1}$ and $s \in\left[2 \varrho^{\hat{j}-(h-1)}, 2 \varrho^{\hat{j}-h}\right]$.

Then the following estimate is also true:

$$
\mu_{h}\left(B_{S}(x)\right) \leq C_{R} s^{k}
$$

for all $x \in B_{1}(0)$ and all $s \in\left[\frac{1}{5} \varrho^{\hat{\jmath}}, \varrho^{\hat{\jmath}-h}\right]$, where $C_{R}$ is the constant appearing in Reifenberg Theorem 4.4.

Proof We proceed in several steps. Let $h \in\{0, \ldots, \hat{\jmath}-1\}$ be fixed.

Step 1 (Application of Reifenberg Theorem) Clearly, if we are able to prove that

$$
\int_{B_{\tau}(w)} \int_{0}^{\tau} \beta_{\mu_{h}}^{k}(y, s)^{2} \frac{d s}{s} d \mu_{h}(y)<\delta_{R} \tau^{k}
$$


holds for all $w \in B_{1}(0)$ and all $\tau<\varrho^{\hat{j}-h}$, then we can exploit the rescaled version of Reifenberg Theorem (Corollary 4.5), and we get exactly the thesis.

Step 2 (Application of the Estimates on $\beta_{\mu}^{k}$ ) Notice that the integral with respect to $\mu_{h}$ appearing in Eq. (6.26) is actually a sum on $y \in \mathcal{C}_{h} \cap B_{\tau}(w)$. Let $y \in \mathcal{C}_{h}$, and consider $\beta_{\mu_{h}}^{k}(y, s)$. Then:

- If $s \leq \frac{1}{5} r_{y}$, then $\beta_{\mu_{h}}^{k}(y, s)=0$, because $y$ is the only point of $\mathcal{C}_{h}$ contained in $B_{s}(y)$ (and thus any $k$-plane through $y$ is a best approximating plane for $\mu_{h}$ );

- If $s \geq \frac{1}{5} r_{y}$, by property 4 of the covering $\mathcal{F}$ (specifically Eq. (6.3)), $u$ is not $\left(\frac{1}{2} \eta, k+1\right)$-invariant in $B_{5 \delta_{0} s}(y)$. Thus we can use Theorem 4.6 with $\bar{r}=5 \delta_{0}$ and $\sigma=5$ (for example!) and obtain:

$$
\beta_{\mu_{h}}^{k}(y, s)^{2} \leq C_{J} s^{-k} \int_{B_{s}(y)}(\theta(z, 5 s)-\theta(z, s)) d \mu_{h}(z)
$$

where now $C_{J}$ depends on $m, p$ and $\eta$ only.

More compactly, if we define the following function:

$$
\begin{aligned}
W(x, r) & \doteq[\theta(x, 5 r)-\theta(x, r)] \chi \mathcal{C}_{h}(x) \chi_{\left(r_{x} / 5, \infty\right)}(r)= \\
& = \begin{cases}\theta(x, 5 r)-\theta(x, r) & \text { if } x \in \mathcal{C}_{h} \text { and } r \geq \frac{1}{5} r_{x} \\
0 & \text { otherwise }\end{cases}
\end{aligned}
$$

then for all $s>0$ (smaller than a suitable constant) we have:

$$
\beta_{\mu_{h}}^{k}(y, s)^{2} \leq C_{J} s^{-k} \int_{B_{s}(y)} W(z, s) d \mu_{h}(z) .
$$

Step 3 By 6.1 and by Tonelli's Theorem, for a fixed $h \in\{0, \ldots, \hat{\jmath}-1\}$ we have:

$$
\begin{aligned}
& \int_{B_{\tau}(w)} \int_{0}^{\tau} \beta_{\mu_{h}}^{k}(y, s)^{2} \frac{d s}{s} d \mu_{h}(y) \\
& \quad \leq \int_{B_{\tau}(w)} \int_{0}^{\tau} C_{J} s^{-k} \int_{B_{s}(y)} W(z, s) d \mu_{h}(z) \frac{d s}{s} d \mu_{h}(y) \leq \\
& \leq \int_{0}^{\tau} C_{J} s^{-k} \int_{B_{\tau}(w)} \int_{B_{s}(y)} W(z, s) d \mu_{h}(z) d \mu_{h}(y) \frac{d s}{s} .
\end{aligned}
$$

Notice that by the triangle inequality

$$
|z-w| \leq|z-y|+|y-w|
$$

so the set

$$
\left\{(y, z) \in \mathbb{R}^{m} \times \mathbb{R}^{m} \mid y \in B_{\tau}(w), z \in B_{S}(y)\right\}
$$


is contained in

$$
\left\{(y, z) \in \mathbb{R}^{m} \times \mathbb{R}^{m} \mid z \in B_{\tau+s}(w), y \in B_{s}(z)\right\}
$$

Using again Tonelli Theorem, we also switch the two integrals in $\mu_{h}$, thus getting:

$$
\begin{aligned}
& \int_{B_{\tau}(w)} \int_{0}^{\tau} \beta_{\mu_{h}}^{k}(y, s)^{2} \frac{d s}{s} d \mu_{h}(y) \\
& \quad \leq \int_{0}^{\tau} C_{J} s^{-k} \int_{B_{\tau+s}(w)} W(z, s)\left(\int_{B_{s}(z)} d \mu_{h}(y)\right) d \mu_{h}(z) \frac{d s}{s} \leq \\
& \leq \int_{0}^{\tau} C_{J} s^{-k} \int_{B_{2 \tau}(w)} W(z, s) \mu_{h}\left(B_{s}(z)\right) d \mu_{h}(z) \frac{d s}{s} .
\end{aligned}
$$

Now for all the relevant pairs $(z, s)$ (for which $W$ is not 0 ) the estimate (6.24) holds:

$$
\int_{B_{\tau}(w)} \int_{0}^{\tau} \beta_{\mu_{h}}^{k}(y, s)^{2} \frac{d s}{s} d \mu_{h}(y) \leq C_{J} C_{f} \int_{B_{2 \tau}(w)} \int_{0}^{\tau} W(z, s) \frac{d s}{s} d \mu_{h}(z) .
$$

Step 4 Let $x \in \mathcal{D}_{\ell}$, so that $r_{x}=\varrho^{\ell}=5^{-\kappa \ell}$ (see the Remark before the statement). The following estimate holds:

$$
\begin{aligned}
& \int_{\frac{1}{5} r_{x}}^{\frac{1}{5}}(\theta(x, 5 s)-\theta(x, s)) \frac{d s}{s} \\
& \quad=\sum_{j=1}^{\kappa \ell} \int_{\left(\frac{1}{5}\right)^{j+1}}^{\left(\frac{1}{5}\right)^{j}} \frac{\theta(x, 5 s)-\theta(x, s)}{s} d s \\
& \quad \leq \sum_{j=1}^{\kappa \ell} \frac{\theta\left(x, 5^{1-j}\right)-\theta\left(x, 5^{-1-j}\right)}{5^{-1-j}}\left(\frac{1}{5}\right)^{j}\left(1-\left(\frac{1}{5}\right)\right) \\
& \quad \leq C\left[\theta(x, 1)-\theta\left(x, 5^{-\kappa \ell}\right)+\theta\left(x, \frac{1}{5}\right)-\theta\left(x, \frac{1}{5} 5^{-\kappa \ell}\right)\right] \\
& \leq C_{7} \zeta,
\end{aligned}
$$

where the last inequality is a consequence of property 4 of the covering $\mathcal{F}$ (specifically Eq. (6.2)) and $C_{7}$ depends on $\varrho$ and $\eta$. Plugging this information into Eq. (6.35) (provided that $\tau \leq \frac{1}{5}$ ), we get

$$
\int_{B_{\tau}(w)} \int_{0}^{\tau} \beta_{\mu_{h}}^{k}(y, s)^{2} \frac{d s}{s} d \mu_{h}(y) \leq C_{J} C_{f} C_{7} \zeta \mu_{h}\left(B_{2 \tau}(w)\right) .
$$


The left hand side is 0 whenever $B_{2 \tau}(w)$ contains a single point of $\mathcal{C}_{h}$; in all the other cases, the assumption (6.24) holds, thus

$$
\int_{B_{\tau}(w)} \int_{0}^{\tau} \beta_{\mu_{h}}^{k}(y, s)^{2} \frac{d s}{s} d \mu_{h}(y) \leq 2^{-k} C_{J} C_{f}^{2} C_{7} \zeta \tau^{k} .
$$

Choosing $\zeta\left(\varrho, C_{f}, m, p, \eta\right) \leq \frac{\delta_{R}(m)}{2^{-k} C_{J} C_{f}^{2} C_{7}}$, we have the desired result.

We can finally prove Proposition 6.4.

Proof of Proposition 6.4 The proof is now a simple induction: by Subtheorem 6.4.2 we have an estimate on $\mu_{0}$ depending on a constant $C_{0}$; applying Subtheorem 6.4.1 the same estimate holds for $\mu_{1}$ with $C_{f}=C_{f}\left(C_{0}, m, p, \varrho\right)$; but then we apply Subtheorem 6.4.3 to improve the constant: the estimate now holds for $\mu_{1}$ with $C_{R}(m)$. So we can repeat the procedure: the final constant for each $\mu_{h}$ will still be $C_{R}(m)$.

\subsection{Second Covering}

The goal now is to refine the covering in order to find balls which satisfy a clean energy drop; that is, we get rid in some sense of the sets of type $\mathcal{H}(x, r)$, where the uniform energy drop does not happen, and which is already bound to lie in the fattening of a $(k-1)$-dimensional plane.

Construction of the second covering Consider again the "first covering" $\mathcal{F}$ for $\mathcal{S}$. It is split in $\mathcal{F}=\mathcal{G} \cup \mathcal{E}$, where $\mathcal{F}, \mathcal{E}$ and $\mathcal{G}$ have the following properties:

1. Balls in $\mathcal{E} \doteq \mathcal{E}^{(0)} \doteq \mathcal{E}_{\hat{\jmath}}$ have radius equal to $r=\varrho^{\hat{\jmath}}$;

2. Balls in $\mathcal{G} \doteq \mathcal{G}_{0} \cup \cdots \cup \mathcal{G}_{\hat{\jmath}-1}$ have radius $\varrho^{h}$ with $h<\hat{\jmath}$; if $B=B_{r}(x) \in \mathcal{G}$, it satisfies the condition

$$
\mathcal{H}_{B}=\left\{y \in B \cap \mathcal{S} \mid \theta\left(y, \frac{1}{5} \varrho r\right)>E-\delta\right\} \subset \mathcal{B}_{\frac{1}{5} \varrho r}\left(V_{B}\right)
$$

for some $(k-1)$-affine subspace $V_{B} \in \mathbf{H}^{k-1}\left(\mathbb{R}^{m}\right)$.

3. The estimate $\sum_{B \in \mathcal{F}} r_{B}^{k} \leq C_{\mathrm{I}}(m)$ holds, where $r_{B}$ is the radius of $B$.

We now refine $\mathcal{F}$ inductively, applying at each step a rescaled version of the procedure from Sect. 6.1.

Step 1 Consider $B \in \mathcal{G}$ and the associated $(k-1)$-plane $V_{B}$. We cover $B \cap \mathcal{S}$ with balls of radius $\varrho r_{B}$, divided in three subcoverings: $\mathcal{E}_{B}$ (with radius equal to $r$ ), $\mathcal{D}_{B}$ (satisfying an energy drop condition), $\mathcal{W}_{B}$ (wild balls on which we have no control).

- If $r_{B}=\varrho^{\hat{\jmath}-1}$, simply cover $B \cap \mathcal{S}$ with at most $C_{8}(m, \varrho)$ balls of radius $\varrho^{\hat{\jmath}}$. Call this covering $\mathcal{E}_{B}$; set $\mathcal{D}_{B}=\mathcal{W}_{B}=\varnothing$. (Actually $C_{8}(m, \varrho)=C(m) \varrho^{-m}$, but it's irrelevant.)

- If $r_{B}>\varrho^{\hat{\jmath}-1}$, we cover $\mathcal{B}_{\frac{1}{5} \varrho r_{B}}\left(\mathcal{H}_{B}\right)$ with at most $C_{9}(m) \varrho^{-(k-1)}$ balls of radius $\varrho r_{B}$; call this covering $\mathcal{W}_{B}$. This is possible since $\mathcal{H}_{B} \subset \mathcal{B}_{\frac{1}{5} \varrho r_{B}}\left(V_{B}\right)$; notice that the case $\mathcal{H}_{B}=\varnothing$ is included. Cover $(B \cap \mathcal{S}) \backslash \mathcal{B}_{\frac{1}{5} e_{B}}\left(\mathcal{H}_{B}\right)$ with at most $C_{8}(m, \varrho)$ 
balls of radius $\varrho r_{B}$; call this covering $\mathcal{D}_{B}$. Set $\mathcal{E}_{B}=\varnothing$. Notice that if $\tilde{B} \in \mathcal{D}_{B}$ then it satisfies the uniform energy drop condition.

At this point we have a covering of $\mathcal{S}$ of this type:

$$
\mathcal{F}^{(1)}=\mathcal{E}^{(1)} \cup \mathcal{D}^{(1)} \cup \mathcal{W}^{(1)},
$$

where

$$
\mathcal{E}^{(1)} \doteq \mathcal{E}^{(0)} \cup \bigcup_{B \in \mathcal{G}} \mathcal{E}_{B}, \quad \mathcal{D}^{(1)} \doteq \bigcup_{B \in \mathcal{G}} \mathcal{D}_{B}, \quad \mathcal{W}^{(1)} \doteq \bigcup_{B \in \mathcal{G}} \mathcal{W}_{B}
$$

and

$$
\begin{aligned}
& \sum_{B \in \mathcal{E}^{(0)}} r_{B}^{k} \leq C_{\mathrm{I}}(m), \quad \sum_{B \in \mathcal{E}^{(1)} \backslash \mathcal{E}^{(0)}} r_{B}^{k} \leq \varrho^{k} C_{8}(m, \varrho) C_{\mathrm{I}}(m) \\
& \sum_{B \in \mathcal{D}^{(1)}} r_{B}^{k} \leq \varrho^{k} C_{8}(m, \varrho) C_{\mathrm{I}}(m), \quad \sum_{B \in \mathcal{W}^{(1)}} r_{B}^{k} \leq C_{9}(m) C_{\mathrm{I}}(m) \varrho^{k} \varrho^{-k+1} .
\end{aligned}
$$

Introduce the constants

$$
\begin{aligned}
K_{1}(\varrho, m) & =C_{\mathrm{I}}(m)\left(1+2 \varrho^{k} C_{8}\right) \\
K_{2}(m) & =C_{\mathrm{I}}(m) C_{9}(m) .
\end{aligned}
$$

so that

$$
\sum_{\mathcal{E}^{(1)} \cup \mathcal{D}^{(1)}} r_{B}^{k} \leq K_{1}, \quad \sum_{\mathcal{W}^{(1)}} r_{B}^{k} \leq K_{2} \varrho
$$

Step $h+1$. Assume that, for some $h \leq \hat{\jmath}-1$, we have a covering of $\mathcal{S}$ of the form $\mathcal{F}^{(h)}=\mathcal{E}^{(h)} \cup \mathcal{D}^{(h)} \cup \mathcal{W}^{(h)}$ with the following properties:

1. If $B \in \mathcal{E}^{(h)}$, then $r_{B}=r=\varrho^{\hat{j}}$;

2. If $B \in \mathcal{D}^{(h)}$, then the energy drop condition holds in $B$;

3. If $B \in \mathcal{W}^{(h)}$, then $\varrho^{\hat{\jmath}}<r_{B} \leq \varrho^{h}$;

4. The estimates

$$
\sum_{B \in \mathcal{E}^{(h)} \cup \mathcal{D}^{(h)}} r_{B}^{k} \leq K_{1} \sum_{j=0}^{h-1}\left(K_{2} \varrho\right)^{j}, \quad \sum_{B \in \mathcal{W}^{(h)}} r_{B}^{k} \leq\left(K_{2} \varrho\right)^{h}
$$

hold true.

Consider a ball $B^{\star} \in \mathcal{W}^{(h)}$. Applying a rescaled version of the first construction (and of Proposition 6.4) we first find a covering $\mathcal{F}_{B^{\star}}$ for $\mathcal{S} \cap B^{\star}$ of the type

$$
\mathcal{F}_{B^{\star}}=\mathcal{G}_{B^{\star} ; h} \cup \cdots \cup \mathcal{G}_{B^{\star} ; \hat{\jmath}-1} \cup \mathcal{E}_{B^{\star} ; \hat{\jmath}}=\mathcal{G}_{B^{\star}} \cup \mathcal{E}_{B^{\star}},
$$


where $\mathcal{G}_{B^{\star}}$ are balls on which the energy drop condition is verified up to a neighborhood of a $(k-1)$-plane, $\mathcal{E}_{B^{\star}}$ are balls of radius $r=\varrho^{\hat{\jmath}}$, and $\sum_{B \in \mathcal{F}_{B^{\star}}} r_{B}^{k} \leq C_{\mathrm{I}}(m) r_{B^{\star}}^{k}$. Secondly, re-cover each ball of $\mathcal{G}_{B^{\star}}$ with a rescaled version of Step 1, thus obtaining

$$
\mathcal{F}_{B^{\star}}^{(h+1)}=\mathcal{E}_{B^{\star}}^{(h+1)} \cup \mathcal{D}_{B^{\star}}^{(h+1)} \cup \mathcal{W}_{B^{\star}}^{(h+1)},
$$

where balls of $\mathcal{E}_{B^{\star}}^{(h+1)}$ have radius $r$, balls of $\mathcal{D}_{B^{\star}}^{(h+1)}$ satisfy the energy drop condition, balls of $\mathcal{W}_{B^{\star}}^{(h+1)}$ have radius $r<r_{B} \leq \varrho^{h+1}$, and

$$
\sum_{B \in \mathcal{E}_{B^{\star}}^{(h+1)} \cup \mathcal{D}_{B^{\star}}^{(h+1)}} r_{B}^{k} \leq K_{1} r_{B^{\star}}^{k}, \quad \sum_{B \in \mathcal{W}_{B^{\star}}^{(h+1)}} r_{B}^{k} \leq K_{2} \varrho r_{B^{\star}}^{k}
$$

Then define

$$
\begin{aligned}
& \mathcal{E}^{(h+1)} \doteq \mathcal{E}^{(h)} \cup \bigcup_{B^{\star} \in \mathcal{W}^{(h)}} \mathcal{E}_{B^{\star}}^{(h+1)}, \quad \mathcal{D}^{(h+1)} \doteq \mathcal{D}^{(h)} \cup \underset{B^{\star} \in \mathcal{W}^{(h)}}{\bigcup} \mathcal{D}_{B^{\star}}^{(h+1)} \\
& \mathcal{W}^{(h+1)} \doteq \bigcup_{B^{\star} \in \mathcal{W}^{(h)}} \mathcal{W}_{B^{\star}}^{(h+1)} \\
& \mathcal{F}^{(h+1)}=\mathcal{E}^{(h+1)} \cup \mathcal{D}^{(h+1)} \cup \mathcal{W}^{(h+1)}
\end{aligned}
$$

All the conditions 1 to 3 are satisfied with $h+1$ instead of $h$; as for the estimates (6.47), we have

$$
\begin{aligned}
\sum_{B \in \mathcal{E}^{(h+1) \cup \mathcal{D}^{(h+1)}}} r_{B}^{k} & =\sum_{B \in \mathcal{E}^{(h)} \cup \mathcal{D}^{(h)}} r_{B}^{k}+\sum_{B^{\star} \in \mathcal{W}^{(h)}} \sum_{B \in \mathcal{E}_{B^{\star}}^{(h+1)} \cup \mathcal{D}_{B^{\star}}^{(h+1)}} r_{B}^{k} \leq \\
& \leq K_{1} \sum_{j=0}^{h-1}\left(K_{2} \varrho\right)^{j}+K_{1} \sum_{B^{\star} \in \mathcal{W}^{(h)}} r_{B^{\star}}^{k} \leq K_{1} \sum_{j=0}^{h}\left(K_{2} \varrho\right)^{j}
\end{aligned}
$$

and

$$
\begin{aligned}
\sum_{B \in \mathcal{W}^{(h+1)}} r_{B}^{k} & =\sum_{B^{\star} \in \mathcal{W}^{(h)}} \sum_{B \in \mathcal{W}_{B^{\star}}^{(h+1)}} r_{B}^{k} \leq \\
& \leq K_{2} \varrho \sum_{B^{\star} \in \mathcal{W}^{(h)}} r_{B^{\star}}^{k} \leq\left(K_{2} \varrho\right)^{h+1} .
\end{aligned}
$$

Thus, as a consequence of this procedure, we have the following.

Proposition 6.5 Let $u \in W^{1, p}(\Omega, \mathcal{N})$ be a p-minimizing map with energy bounded by $\Lambda$. Let $\eta>0$ be a constant and $1 \leq k \leq m$. Assume that $E \leq \Lambda$ is such that $\theta(y, 1) \leq E$ for all $y \in B_{1}(0) \cap \mathcal{S}$. Let $\mathcal{S} \subset \mathcal{S}_{\eta, \delta_{0} r}^{k}(u)$ for some $r>0$. There exists a finite covering $\mathcal{F}^{\star}$ of $\mathcal{S}$ with the following properties: 
(i) All the radii satisfy $r_{B} \geq \bar{\varrho} r$, where $\bar{\varrho} \in(0,1)$ is a constant only depending on $m$;

(ii) The $k^{\text {th }}$ powers of the radii are controlled by $\sum_{B \in \mathcal{F} \star} r_{B}^{k} \leq C_{I I}$, where $C_{I I}$ depends only on $m$.

(iii) $\mathcal{F}^{\star}=\mathcal{L}^{\star} \cup \mathcal{D}^{\star}$, where:

(A) For all $B \in \mathcal{L}^{\star}, r_{B} \leq r$ (that is, $r_{B}$ is lower than or equal to the needed radius);

(B) Every ball $B \in \mathcal{D}^{\star}$ satisfies a uniform energy drop condition:

$$
\theta\left(y, \frac{1}{5} r_{B}\right)<E-\delta \quad \text { for all } y \in \mathcal{S} \cap B
$$

Here both $\delta_{0}$ and $\delta$ are constants that depend on $m, p, \mathcal{N}, \Lambda, \eta$ (and nothing else).

Proof Let $\bar{\varrho}=\bar{\varrho}(m)=\frac{1}{2} K_{2}(m)^{-1}$, where $K_{2}$ is the constant introduced in (6.45). Once $\bar{\varrho}(m)$ is chosen, also a constant $\bar{\zeta}(m, p, \eta)$ is fixed by Subtheorem 6.4.3; as a consequence, $\delta_{0}(m, p, \eta)$ gets determined by Lemma 5.1 and the constant $\delta(m, p, \eta)$ is fixed as well by Lemma 5.1 and Lemma 5.2. Assume that $\bar{\varrho}^{\hat{\jmath}} \leq r<\bar{\varrho}^{\hat{\jmath}-1}$ for some $\hat{\jmath} \in \mathbb{N}$. Perform the construction (of the first covering and then) of the second covering until Step $\hat{\jmath}$. Then:

- $\mathcal{W}^{(\hat{\jmath})}=\varnothing$ (by the bounds on the radii), so $\mathcal{F}^{(\hat{\jmath})}=\mathcal{E}^{(\hat{\jmath})} \cup \mathcal{D}^{(\hat{\jmath})}$;

- The balls in $\mathcal{E}^{(\hat{\jmath})}$ have radius $\bar{\varrho}^{\hat{\jmath}} \leq r$;

- The balls in $\mathcal{D}^{(\hat{\jmath})}$ satisfy the energy drop condition;

- Each ball in $\mathcal{F}^{(\hat{\jmath})}$ has radius greater than or equal to $\bar{\varrho}^{\hat{\jmath}} \geq \bar{\varrho} r$.

Moreover, by Eq. (6.47),

$$
\sum_{B \in \mathcal{F}^{(\hat{\jmath})}} r_{B}^{k} \leq K_{1}(m, \bar{\varrho}(m)) \sum_{j=0}^{\hat{\jmath}-1}\left(K_{2} \varrho\right)^{j} \leq K_{3}(m) \sum_{h=0}^{\infty}\left(\frac{1}{2}\right)^{h} \leq 2 K_{3}(m) .
$$

This proves the proposition, by setting $C_{\mathrm{II}}(m)=2 K_{3}(m), \mathcal{L}^{\star}=\mathcal{E}^{(\hat{\jmath})}, \mathcal{D}^{\star}=\mathcal{D}^{(\hat{\jmath})}$

\section{Proof of the Main Theorems}

We split the main result Theorem 3.7 in two parts, one concerning the estimate on the volume $\operatorname{Vol}\left(\mathcal{B}_{r}\left(\mathcal{S}_{\eta, \delta_{0} r}^{k}(u)\right) \cap B_{1}(0)\right)$, and one for the rectifiability of $\mathcal{S}_{\eta}^{k}$.

\subsection{Volume Estimate}

Theorem 7.1 Let $u \in W^{1, p}(\Omega, \mathcal{N})$ be a p-minimizing map with energy bounded by $\Lambda$. Let $\eta>0$ and $1 \leq k \leq m$. There exists a constant $C_{1}=C_{1}(m, \mathcal{N}, p, \Lambda, \eta)$ such that for any $r>0$

$$
\operatorname{Vol}\left(\mathcal{B}_{r}\left(\mathcal{S}_{\eta, \delta_{0} r}^{k}(u)\right) \cap B_{1}(0)\right) \leq C_{1} r^{m-k}
$$


The proof is a straightforward consequence of the following lemma:

Lemma 7.2 Let $m, p, \Lambda, \eta, k$ be constants, $u$ a map and $r>0$ as in Theorem 7.1. For any number $i \in \mathbb{N}$ there exists a covering $\mathcal{F}_{i}^{\star}$ of the set $\mathcal{S} \doteq \mathcal{S}_{\eta, \delta_{0} r}^{k}(u) \cap B_{1}(0)$ consisting of open balls, satisfying the following properties:

(i) The radii $r_{B}$ satisfy

$$
\sum_{B \in \mathcal{F}_{i}^{\star}} r_{B}^{k} \leq\left(c_{7}(m) C_{I I}(m)\right)^{i}
$$

for some new dimensional constant $c_{7}(m)$ and the old constant $C_{I I}(m)$ coming from Proposition 6.5;

(ii) $\mathcal{F}_{i}^{\star}=\mathcal{L}_{i}^{\star} \cup \mathcal{D}_{i}^{\star}$, where:

(A) For all $B \in \mathcal{L}_{i}^{\star}$, $\varrho r \leq r_{B} \leq r$, where $\bar{\varrho}=\bar{\varrho}(m) \in(0,1)$ is the constant introduced in Proposition 6.5;

(B) For all $B \in \mathcal{D}_{i}^{\star}$ and all $y \in \mathcal{S} \cap B$, we have

$$
\theta\left(y, \frac{1}{5} r_{B}\right) \leq \Lambda-i \delta
$$

where $\delta=\delta(m, p, \Lambda, \mathcal{N}, \eta)$ is the constant determined by Proposition 6.5.

Proof We proceed by induction on $i \in \mathbb{N}$. For $i=0$, we can simply take $\mathcal{F}_{0}^{\star}=\mathcal{D}_{0}^{\star}=$ $\left\{B_{1}(0)\right\}$. Assume then the lemma is true for some $i \geq 0$. Consider a ball $B_{0} \in \mathcal{D}_{i}^{\star}$, and cover it with $c_{7}(m)$ balls of radius $\frac{1}{5} r_{B_{0}}$ (call $\mathcal{D}_{i, B_{0}}$ this covering); for each of these balls $B$ consider the rescaling of $B$ (and $u$ ) through the transformation that maps it into the unit ball. Applying Proposition 6.5 with $E=\Lambda-i \delta$ and $\mathcal{S} \backslash \bigcup_{j=1}^{i} \mathcal{L}_{j}$, and scaling back to the original $B$, we find a covering $\mathcal{F}_{B}^{\star}=\mathcal{L}_{B}^{\star} \cup \mathcal{D}_{B}^{\star}$ satisfying:

1. If $\tilde{B} \in \mathcal{L}_{B}^{\star}$, then $\bar{\varrho} r \leq r_{\tilde{B}} \leq r$;

2. If $\tilde{B} \in \mathcal{D}_{B}^{\star}$, then $\theta\left(y, \frac{1}{5} r_{\tilde{B}}\right) \leq \Lambda-i \delta-\delta$ for all $y \in \tilde{B} \cap \mathcal{S}$;

3. $\sum_{\tilde{B} \in \mathcal{F}_{B}^{\star}} r_{\tilde{B}}^{k} \leq C_{\mathrm{II}} r_{B}^{k}$.

Thus, by defining

$$
\begin{aligned}
& \mathcal{D}_{i+1}^{\star} \doteq \bigcup_{B_{0} \in \mathcal{D}_{i}^{\star}} \bigcup_{B \in \mathcal{D}_{B_{0}, i}} \mathcal{D}_{B}^{\star} \\
& \mathcal{L}_{i+1}^{\star} \doteq \mathcal{L}_{i}^{\star} \cup \bigcup_{B_{0} \in \mathcal{D}_{i}^{\star}} \bigcup_{B \in \mathcal{D}_{B_{0}, i}} \mathcal{L}_{B}^{\star},
\end{aligned}
$$

we get the needed result.

Proof of Theorem 7.1 We apply Lemma 7.2 with $\hat{\imath}(m, p, \Lambda, \mathcal{N}, \eta) \doteq\left\lfloor\frac{\Lambda}{\delta}\right\rfloor+1$, where $\delta=\delta(m, p, \Lambda, \mathcal{N}, \eta)$ is the constant determined by Proposition 6.5. Consider the covering $\mathcal{F}^{\dagger} \doteq \mathcal{F}_{\hat{\imath}}^{\star}$ of $\mathcal{S}_{\eta, \delta_{0} r}^{k}(u) \cap B_{1}(0)$ : the energy drop condition $\theta\left(y, \frac{1}{5} r_{B}\right) \leq$ 
$\Lambda-\hat{\imath} \delta<0$ can never hold, since by definition the normalized energy $\theta$ is nonnegative. Thus $\mathcal{F}^{\dagger}$ consists of a collection of balls satisfying

$$
\begin{aligned}
& \sum_{B \in \mathcal{F}^{\dagger}} r_{B}^{k} \leq\left(c_{7}(m) C_{\mathrm{II}}(m)\right)^{\hat{\imath}} \doteq C_{\mathrm{III}}(m, p, \Lambda, \mathcal{N}, \eta), \\
& \bar{\varrho} r \leq r_{B} \leq r \text { for any } B \in \mathcal{F}^{\dagger} .
\end{aligned}
$$

By elementary geometric properties, the collection of balls $\left\{B_{2 r}(x) \mid B_{r_{B}}(x) \in \mathcal{F}^{\dagger}\right\}$ covers the tubular neighborhood $\mathcal{B}_{r}\left(\mathcal{S}_{\eta, \delta_{0} r}^{k}(u)\right) \cap B_{1}(0)$. In particular, by Eqs. (7.5) and (7.6):

$$
\begin{aligned}
\operatorname{Vol}\left(\mathcal{B}_{r}\left(\mathcal{S}_{\eta, \delta_{0} r}^{k}(u)\right) \cap B_{1}(0)\right) & \leq \sum_{B \in \mathcal{F}^{\dagger}} \omega_{m}(2 r)^{m} \leq \omega_{m} 2^{m} r^{m-k} \sum_{B \in \mathcal{F}^{\dagger}}\left(\frac{r_{B}}{\bar{\varrho}}\right)^{k} \leq \\
& \leq \frac{\omega_{m} 2^{m} C_{\mathrm{III}}}{\bar{\varrho}^{k}} r^{m-k},
\end{aligned}
$$

where $\bar{\varrho}=\bar{\varrho}(m)$ and $C_{\mathrm{III}}=C_{\mathrm{III}}(m, p, \Lambda, \mathcal{N}, \eta)$. By defining

$$
C_{1}(m, \mathcal{N}, p, \Lambda, \eta) \doteq \frac{\omega_{m} 2^{m} C_{\mathrm{III}}}{\bar{\varrho}^{k}},
$$

the result is proved.

\subsection{Rectifiability}

We now tackle the problem of the rectifiability of the strata of type $\mathcal{S}_{k}^{\eta}$. It is clear that we will need to use the second part of Theorem 4.4; the technique is basically the same we used for the volume estimates, even with some simplifications.

Theorem 7.3 Let $u \in W^{1, p}(\Omega, \mathcal{N})$ be a p-energy minimizing map. For any $\eta>0$ and any $0 \leq k \leq m$, the stratum $\mathcal{S}_{\eta}^{k}(u)$ is $k$-rectifiable.

As we will see shortly, the result follows easily from this Lemma.

Lemma 7.4 Let $m, p, \Lambda, \eta$ be fixed. There exist a universal constant $\kappa(m, p, \Lambda, \mathcal{N}, \eta)$ with $0<\kappa<1$ such that the following holds. Let $u$ be $p$-minimizing, and let $\mathcal{S} \subset$ $\mathcal{S}_{\eta}^{k}(u) \cap B_{1}(0)$ be a $\mathscr{H}^{k}$-measurable subset. There exists a $\mathscr{H}^{k}$-measurable subset $\mathcal{R} \subset \mathcal{S}$ with the following properties:

1. $\mathscr{H}^{k}(\mathcal{R}) \leq \kappa \mathscr{H}^{k}(\mathcal{S})$;

2. The set $\mathcal{S} \backslash \mathcal{R}$ is $k$-rectifiable.

Before proving this lemma, which requires some effort, we show how it is applied to prove Theorem 7.3.

Proof of Theorem 7.3 By the definition of $k$-rectifiability, it is sufficient to prove the statement for $\mathcal{S}_{\eta}^{k}(u) \cap \Omega_{j}$, where $\left\{\Omega_{j}\right\}_{j}$ is an increasing sequence of compact sets exhausting $\Omega$; by taking a finite covering of each $\Omega_{j}$ with balls contained in $\Omega$ and 
then rescaling, it is actually enough to prove the $k$-rectifiability of $\mathcal{S}_{\eta}^{k}(u) \cap B_{1}(0)$. Notice that by Theorem 7.1 the (upper) $k$-dimensional Minkowski content of $\mathcal{S}_{\eta}^{k}(u) \cap B_{1}(0)$ is finite, since $\mathcal{S}_{\eta}^{k}(u) \subset \mathcal{S}_{\eta, r}^{k}(u)$ for any $r>0$; thus $\mathscr{H}^{k}\left(\mathcal{S}_{\eta}^{k}(u) \cap B_{1}(0)\right)$ is also finite. that:

By induction, for any $j \in \mathbb{N}$ there exists a $\mathscr{H}^{k}$-measurable set $\mathcal{R}_{j} \subset \mathcal{S}_{\eta}^{k}(u)$ such

- $\mathscr{H}^{k}\left(\mathcal{R}_{j}\right) \leq \kappa^{j} \mathscr{H}^{k}\left(\mathcal{S}_{\eta}^{k}(u) \cap B_{1}(0)\right)$;

- The set $\left(\mathcal{S}_{\eta}^{k}(u) \cap B_{1}(0)\right) \backslash \mathcal{R}_{j}$ is $k$-rectifiable.

This is easily proved: the step $j=1$ comes from the application of Lemma 7.4 to the stratum $\mathcal{S}_{\eta}^{k}(u) \cap B_{1}(0)$, while the $(j+1)^{\text {th }}$ step descends from the application of the same Lemma to $\mathcal{R}_{j}$. Now we can define

$$
\begin{aligned}
& \tilde{\mathcal{R}} \doteq \bigcap_{j \in \mathbb{N}} \mathcal{R}_{j} \\
& \tilde{\mathcal{S}} \doteq\left(\mathcal{S}_{\eta}^{k}(u) \cap B_{1}(0)\right) \backslash \tilde{\mathcal{R}}=\bigcup_{j \in \mathbb{N}}\left(\left(\mathcal{S}_{\eta}^{k}(u) \cap B_{1}(0)\right) \backslash \mathcal{R}_{j}\right) .
\end{aligned}
$$

Here $\tilde{\mathcal{R}}$ has $\mathscr{H}^{k}$-measure zero; and $\tilde{\mathcal{S}}$ is the countable union of sets, each of which is countable union of Lipschitz $k$-graphs; therefore $\tilde{\mathcal{S}}$ itself is a countable union of Lipschitz $k$-graphs. This means precisely that $\mathcal{S}_{\eta}^{k}(u) \cap B_{1}(0)$ is $k$-rectifiable.

Now we turn to prove Lemma 7.4.

Proof We can assume that $\mathscr{H}^{k}(\mathcal{S})>0$, otherwise the statement is trivial.

Step 1 Consider the following map: for $x \in B_{1}(0)$ and $r>0$ (small enough),

$$
f_{r}(x) \doteq \theta(x, r)-\theta(x, 0),
$$

where $\theta(x, 0) \doteq \lim _{s \rightarrow 0} \theta(x, s)$. As $r$ tends to 0 , the map $f_{r}$ converges pointwise and decreasingly to the constant function $f_{0} \equiv 0$; moreover, all the maps $f_{r}$ are bounded by the constant map $\Lambda$, which is integrable with respect to the measure $\mathscr{H}^{k}\llcorner\mathcal{S}$ by Theorem 7.1. Now fix a $\delta>0$. By the Dominated Convergence Theorem, there exists a $\bar{r}>0$ depending on $\delta$ such that

$$
\int_{\mathcal{S}} f_{5 \bar{r}}(x) d \mathscr{H}^{k}(x) \leq \delta^{2} \mathscr{H}^{k}(\mathcal{S})
$$

Consider the following sets:

$$
\begin{aligned}
F_{\delta} & \doteq\left\{x \in \mathcal{S} \mid f_{5 \bar{r}(\delta)}(x)>\delta\right\} \\
G_{\delta} & \doteq\left\{x \in \mathcal{S} \mid f_{5 \bar{r}(\delta)}(x) \leq \delta\right\}=\mathcal{S} \backslash F_{\delta}
\end{aligned}
$$

observe that, since $f_{5 \bar{r}}$ is nonnegative, we have:

$$
\int_{\mathcal{S}} f_{5 \bar{r}}(x) d \mathscr{H}^{k}(x) \geq \int_{F_{\delta}} f_{5 \bar{r}}(x) d \mathscr{H}^{k}(x) \geq \delta \mathscr{H}^{k}\left(F_{\delta}\right)
$$


this, combined with Eq. (7.12), gives

$$
\mathscr{H}^{k}\left(F_{\delta}\right) \leq \delta \mathscr{H}^{k}(\mathcal{S})
$$

We claim that, for $\delta$ sufficiently small, the set $G_{\delta}$ is $k$-rectifiable; if we manage to show this, then the lemma is proved. In order to prove this claim, we consider a finite covering $\left\{B_{\bar{r}}\left(x_{i}\right)\right\}_{i=1}^{L}$ of $G_{\delta}$ made with balls of the fixed radius $\bar{r}(\delta)$. It is sufficient to show that for $\delta$ small $G_{\delta} \cap B_{\bar{r}(\delta)}\left(x_{i}\right)$ is rectifiable for any $i$ : our main aim will be now to check the applicability of the second Reifenberg Theorem (Theorem 4.4, part (ii)), that gives exactly that result.

Step 2 Fix a ball $B_{\bar{r}(\delta)}\left(x_{i}\right)$, with $i \in\{1, \ldots, L\}$, and apply the usual transformation $\lambda_{x_{i}, \bar{r}}^{-1}$. We set

$$
\tilde{u}=T_{x_{i}, \bar{r}} u, \quad \tilde{G}_{\delta}=\lambda_{x_{i}, \bar{r}}^{\leftarrow}\left(G_{\delta}\right) \cap B_{1}(0)
$$

Also, we define $\mu_{\delta}$ to be the measure $\mathscr{H}^{k}\left\llcorner\tilde{G}_{\delta}\right.$ on the unit ball $B_{1}(0)$. Notice that for any $x \in \tilde{G}_{\delta}$ we have:

$$
\theta^{\tilde{u}}(x, 5)-\theta^{\tilde{u}}(x, 0) \leq \delta,
$$

by the definition of $G_{\delta}$ and the usual scale invariance properties of $\theta$. Now the original $G_{\delta}$ was a subset of $\mathcal{S}_{\eta}^{k}(u)$, hence $u$ was not $(\eta, k+1)$-invariant in $B_{\bar{r} s}(x)$ for any point $x \in G_{\delta}$ and for any $s>0$; consequently, for any point $x$ in the transformed set $\tilde{G}_{\delta}$ and for any $s>0, \tilde{u}$ is not $(\eta, k+1)$-invariant in $B_{s}(x)$. This is what we need to apply Theorem 4.6 on any ball $B_{S}(x)$; and we apply it to the finite measure $\mu_{\delta}=\mathscr{H}^{k}\left\llcorner\tilde{G}_{\delta}\right.$. We obtain that, for any $x \in \tilde{G}_{\delta}$ and any $0<s \leq 1$,

$$
\beta_{\tilde{G}_{\delta}}^{k}(x, s)^{2} \leq C_{10}(m, p, \eta) s^{-k} \int_{B_{s}(x)} \theta(y, 5 s)-\theta(y, s) d \mu_{\delta}(y) .
$$

This goes in the direction we need, since we are trying to check if Reifenberg condition ( $k$-Reif) is satisfied. Following what we did in the proof of Proposition 6.4, we first fix $w \in B_{1}(0)$ and $r \leq 1$; for all $0<s \leq r$ we compute:

$$
\begin{aligned}
& \int_{B_{r}(w)} \beta_{\tilde{G}_{\delta}}^{k}(x, s)^{2} d \mu_{\delta}(x) \\
& \quad \leq C_{10} s^{-k} \int_{B_{r}(w)}\left(\int_{B_{s}(x)}\left[\theta^{\tilde{u}}(y, 5 s)-\theta^{\tilde{u}}(y, s)\right] d \mu_{\delta}(y)\right) d \mu_{\delta}(x)
\end{aligned}
$$

Observe that we are allowed to do this since $\mu_{\delta}$ is supported in $\tilde{G}_{\delta}$. As we have already 
noticed in Proposition 6.4, if $|x-w|<r$ and $|y-x|<s$, then $|y-w|<r+s$ : thus we can estimate

$$
\begin{aligned}
& \int_{B_{r}(w)} \beta_{\tilde{G}_{\delta}}^{k}(x, s)^{2} d \mu_{\delta}(x) \\
& \quad \leq C_{10} s^{-k} \int_{B_{r+s}(w)} \int_{B_{s}(y)}\left[\theta^{\tilde{u}}(y, 5 s)-\theta^{\tilde{u}}(y, s)\right] d \mu_{\delta}(x) d \mu_{\delta}(y) \\
& \leq C_{10} s^{-k} \int_{B_{r+s}(w)}\left[\theta^{\tilde{u}}(y, 5 s)-\theta^{\tilde{u}}(y, s)\right] \mathscr{H}^{k}\left(\tilde{G}_{\delta} \cap B_{s}(y)\right) d \mu_{\delta}(y) .
\end{aligned}
$$

But now we can exploit the uniform volume estimates given by Theorem 7.1 (appropriately rescaled); we get the following uniform a priori upper bound:

$$
\mathscr{H}^{k}\left(\lambda_{x_{i}, \bar{r}}^{\leftarrow}\left(\mathcal{S}_{\eta}^{k}(u)\right) \cap B_{s}(y)\right) \leq C_{1}(m, p, \mathcal{N}, \Lambda, \eta) s^{k}
$$

notice that thanks to this a priori estimate it is not necessary to reproduce the induction argument of Proposition 6.4. Plugging this information in the previous inequality we get:

$$
\int_{B_{r}(w)} \beta_{\tilde{G}_{\delta}}^{k}(x, s)^{2} d \mu_{\delta}(x) \leq C_{10} C_{1} \int_{B_{r+s}(w)}\left[\theta^{\tilde{u}}(y, 5 s)-\theta^{\tilde{u}}(y, s)\right] d \mu_{\delta}(y) .
$$

In order to check the validity of Eq. ( $k$-Reif), we now consider the left hand side of that inequality: applying Tonelli Theorem (twice), we find:

$$
\begin{aligned}
& \int_{B_{r}(w)}\left(\int_{0}^{r} \beta_{\tilde{G}_{\delta}}^{k}(x, s)^{2} \frac{d s}{s}\right) d \mu_{\delta}(x)=\int_{0}^{r}\left(\int_{B_{r}(w)} \beta_{\tilde{G}_{\delta}}^{k}(x, s)^{2} d \mu_{\delta}(x)\right) \frac{d s}{s} \\
& \leq C_{10} C_{1} \int_{0}^{r}\left(\int_{B_{2 r}(w)}\left[\theta^{\tilde{u}}(y, 5 s)-\theta^{\tilde{u}}(y, s)\right] d \mu_{\delta}(y)\right) \frac{d s}{s} \\
& =C_{10} C_{1} \int_{B_{2 r}(w)}\left(\int_{0}^{r}\left[\theta^{\tilde{u}}(y, 5 s)-\theta^{\tilde{u}}(y, s)\right] \frac{d s}{s}\right) d \mu_{\delta}(y) .
\end{aligned}
$$

Consider for a moment the inner integral; $r$ can simply be bounded by 1 . We use basically the same trick we exploited in Proposition 6.4: 


$$
\begin{aligned}
& \int_{0}^{1}\left[\theta^{\tilde{u}}(y, 5 s)-\theta^{\tilde{u}}(y, s)\right] \frac{d s}{s} \\
& \quad=\sum_{j=0}^{\infty} \int_{5^{-(j+1)}}^{5^{-j}} \frac{\theta^{\tilde{u}}(y, 5 s)-\theta^{\tilde{u}}(y, s)}{s} d s \leq \\
& \quad \leq \sum_{j=0}^{\infty} \int_{5^{-(j+1)}}^{5^{-j}} \frac{\theta^{\tilde{u}}\left(y, 5^{-j+1}\right)-\theta^{\tilde{u}}\left(y, 5^{-j-1}\right)}{5^{-j-1}} d s \leq \\
& \leq C_{10} \sum_{j=0}^{\infty}\left[\theta^{\tilde{u}}\left(y, 5^{-j+1}\right)-\theta^{\tilde{u}}\left(y, 5^{-j-1}\right)\right] \leq \\
& \leq C_{10}\left[\left(\theta^{\tilde{u}}(y, 5)-\theta^{\tilde{u}}(y, 0)\right)+\left(\theta^{\tilde{u}}(y, 1)-\theta^{\tilde{u}}(y, 0)\right)\right] \leq \\
& \leq 2 C_{10} \delta .
\end{aligned}
$$

Therefore we can insert this piece of information in the previous integral; using again the upper bound (7.22) on the measure of the singular stratum, we find, for a new constant $C_{13}(m, p, \mathcal{N}, \Lambda, \eta)$ :

$$
\begin{aligned}
\int_{B_{r}(w)}\left(\int_{0}^{r} \beta_{\tilde{G}_{\delta}}^{k}(x, s)^{2} \frac{d s}{s}\right) d \mu_{\delta}(x) & \leq C_{12} \mu_{\delta}\left(B_{2 r}(w)\right) \delta \leq \\
& \leq C_{13} \delta r^{k}
\end{aligned}
$$

Taking

$$
\delta<\frac{\delta_{\mathrm{R}}(m)}{C_{13}(m, p, \mathcal{N}, \Lambda, \eta)},
$$

we get exactly the hypothesis needed for the second part of Reifenberg Theorem: thus $\tilde{G}_{\delta}$ is $k$-rectifiable, and tracing back the steps of the proof this proves the $k$-rectifiability of $G_{\delta}$.

Acknowledgements I am deeply grateful to Daniele Valtorta for the precious suggestions he gave me while writing this article; and before that, for sharing his knowledge and ideas about this topic.

Funding Open Access funding provided by Università degli Studi di Trento

Open Access This article is licensed under a Creative Commons Attribution 4.0 International License, which permits use, sharing, adaptation, distribution and reproduction in any medium or format, as long as you give appropriate credit to the original author(s) and the source, provide a link to the Creative Commons licence, and indicate if changes were made. The images or other third party material in this article are included in the article's Creative Commons licence, unless indicated otherwise in a credit line to the material. If material is not included in the article's Creative Commons licence and your intended use is not permitted by statutory regulation or exceeds the permitted use, you will need to obtain permission directly from the copyright holder. To view a copy of this licence, visit http://creativecommons.org/licenses/by/4.0/. 


\section{References}

1. Azzam, J., Tolsa, X.: Characterization of $n$-rectifiability in terms of Jones' square function: Part II. Geom. Funct. Anal. 25(5), 1371-1412 (2015)

2. Cheeger, J., Naber, A.: Quantitative stratification and the regularity of harmonic maps and minimal currents. Comm. Pure Appl. Math. 66(6), 965-990 (2013)

3. David, G., Toro, T.: Reifenberg parameterizations for sets with holes. Mem. Am. Math. Soc., 215(1012), vi+102 (2012)

4. Edelen, N., Naber, A., Valtorta, D.: Quantitative reifenberg theorem for measures. arXiv preprint arXiv:1612.08052, (2016)

5. Edelen, N., Naber, A., Valtorta, D.: Effective Reifenberg theorems in Hilbert and Banach spaces. Math. Ann. 374(3-4), 1139-1218 (2019)

6. Hardt, R., Lin, F.-H.: Mappings minimizing the $L^{p}$ norm of the gradient. Comm. Pure Appl. Math. 40(5), 555-588 (1987)

7. Hirsch, J., Stuvard, S., Valtorta, D.: Rectifiability of the singular set of multiple-valued energy minimizing harmonic maps. Trans. Amer. Math. Soc. 371(6), 4303-4352 (2019)

8. Jones, P.W.: Rectifiable sets and the traveling salesman problem. Invent. Math. 102(1), 1-15 (1990)

9. Luckhaus, S.: Partial Hölder continuity for minima of certain energies among maps into a Riemannian manifold. Indiana Univ. Math. J. 37(2), 349-367 (1988)

10. Miśkiewicz, M.: Discrete Reifenberg-type theorem. Ann. Acad. Sci. Fenn. Math. 43(1), 3-19 (2018)

11. Moser, R.: Partial Regularity for Harmonic Maps and Related Problems. World Scientific Publishing Co. Pte. Ltd., Hackensack, NJ, (2005)

12. Nash, J.: $C^{1}$ isometric imbeddings. Ann. Math. 2(60), 383-396 (1954)

13. Naber, A., Valtorta, D.: Rectifiable-Reifenberg and the regularity of stationary and minimizing harmonic maps. Ann. Math. (2) 185(1), 131-227 (2017)

14. Naber, A., Valtorta, D.: Stratification for the singular set of approximate harmonic maps. Math. Z. 290(3-4), 1415-1455 (2018)

15. Naber, A., Valtorta, D., Veronelli, G.: Quantitative regularity for $p$-harmonic maps. Commun. Anal. Geom. 27(1), 111-159 (2019)

16. Pajot, H.: Analytic capacity, rectifiability, Menger curvature and the Cauchy integral. Lecture Notes in Mathematics, vol. 1799. Springer, Berlin (2002)

17. Schoen, R.M.: Analytic aspects of the harmonic map problem. In: Seminar on Nonlinear Partial Differential Equations. pp. 321-358. Springer, (1984)

18. Simon, L.: Theorems on regularity and singularity of energy minimizing maps. Lectures in Mathematics ETH Zürich. Birkhäuser Verlag, Basel, (1996). Based on lecture notes by Norbert Hungerbühler

19. Schoen, R., Uhlenbeck, K.: A regularity theory for harmonic maps. J. Differ. Geom. 17(2), 307-335 (1982)

20. Toro, T.: Geometric conditions and existence of bi-Lipschitz parameterizations. Duke Math. J. 77(1), 193-227 (1995)

21. Xin, Y.: Geometry of harmonic maps. Progress in Nonlinear Differential Equations and their Applications, vol. 23. Birkhäuser Boston Inc., Boston, MA (1996)

Publisher's Note Springer Nature remains neutral with regard to jurisdictional claims in published maps and institutional affiliations. 